\title{
Arctiini Leach, [1815] (Lepidoptera, Erebidae, Arctiinae) of the Brazilian Amazon. I-Subtribe Phaegopterina Kirby, 1892
}

\author{
José A. Teston $^{1 *}$ and Viviane G. Ferro ${ }^{2}$ \\ 1 Universidade Federal do Oeste do Pará (UFOPA), Programa de Pós-Graduação em Recursos Naturais da Amazônia (PPGRNA) e Instituto \\ de Ciências da Educação (ICED) - Laboratório de Estudos de Lepidópteros Neotropicais (LELN). Rua Vera Paz s/n, CEP 68040-255, \\ Santarém, PA, Brazil \\ 2 Universidade Federal de Goiás (UFG), Instituto de Ciências Biológicas, Departamento de Ecologia. Caixa Postal 131, CEP 74001-970, \\ Goiânia, GO, Brazil \\ * Corresponding author. E-mail: jateston@ufpa.br; jateston@gmail.com
}

\begin{abstract}
This study aims to identify and record Arctiini specimens from the Brazilian Amazon, as well as upgrade the existing lists regarding this tribe. The material included in this study consisted of specimens from collections, and insects collected in the field, as well as a literature review,. There are 469 species of Phaegopterina, including 52 are new recorded species, for the Brazilian Amazon.
\end{abstract}

Key words: Amazon; inventory; Noctuoidea; tiger moths

\section{INTRODUCTION}

Moths of the subfamily Arctiinae have a wide geographical distribution. There are approximately 6,000 species in the Neotropics (Heppner 1991). There are 1,391 recorded species of Arctiinae in Brazil and 753 species in the Brazilian Amazon (Ferro and Diniz 2010). Species of Arctiinae are distributed in the tribes Arctiini, Amerilini, Lithosiini, and Syntomini (Zahiri et al. 2012). The tribe Arctiini has six subtribes: Callimorphina, Ctenuchina, Euchromiina, Pericopina, Phaegopterina, and Spilosomina (Vincent and Laguerre 2014). Species of Phaegopterina are nocturnal, have medium size, a wing span of $20-90 \mathrm{~mm}$, a robust abdomen, triangular forewings, and hind wings that are wide and rounded (Costa Lima 1950; Piñas Rubio et al. 2000; Jacobson and Weller 2002). Some phaegopterine species exhibit sexual dimorphism. Many have aposematic coloring on the wings and on the entire body and the abdomen often presents alternating bands of colors (Piñas Rubio et al. 2000). They possess a pair of tympanic organs located on either side of the metathorax above the spiracles (Costa Lima 1950). Some caterpillars sequester pyrrolizidine alkaloids of their host plants and use them in defense and in the synthesis of pheromones (Kitching and Rawlins 1999). They are found worldwide, but mainly in the Neotropics, where there are 1,720 known species (Vincent and Laguerre 2014).

The Amazon biome extends from the Atlantic Ocean to the eastern slopes of the Andes, to approximately 600 $m$ (Ab'Saber 1977). It covers part of nine countries in South America, with $69 \%$ of this area belonging to Brazil (Vieira et al. 2008). Currently, this biome has suffered from logging, deforestation, fires, fragmentation, mining, wildlife extinction, the invasion of alien species, and wildlife trafficking (Fearnside 2003).

We present a list of phaegopterine moths existing in the Brazilian Amazon. We built the species list mainly from specimens deposited in the most important Brazilian collections, a literature review, and field samples.

\section{MATERIALS AND METHODS}

We carried out intensive literature searches, website of Departament of Entomology Collections of Smithsonian National Museum of Natural History (http:// collections.nmnh.si.edu/search/ento), and examined the entomological collections of the Instituto Nacional de Pesquisas na Amazônia (INPA) in Manaus, Museu Paraense Emilio Goeldi (MPEG) in Belém, Coleção Becker (VOB) in Camacan, Coleção Entomológica Padre Jesus Santiago Moure of the Universidade Federal do Paraná (DZUP) in Curitiba, Fundação Instituto Oswaldo Cruz (FIOC) in Rio de Janeiro, Museu de Zoologia of the Universidade de São Paulo (MZUSP) in São Paulo, Museu Nacional of the Universidade Federal do Rio de Janeiro (MNRJ) in Rio de Janeiro, and Laboratório de Estudos de Lepidópteros Neotropicais (LELN) of the Universidade Federal do Oeste do Pará (UFOPA) in Santarém. To identify the species, we used the literature (Hampson 1901, 1920; Seitz 1919-1925; Watson 1971, 1973, 1975, 
1980) and comparative material in collections. The systematic organization to generic level follows Coenen and Gibeaux (2014a, 2014b) and Vincent and Laguerre (2014).

The list is organized alphabetically. Species without precise location (such as Mato Grosso and Maranhão states), and also that occurred in locations that belong to two biomes (e.g., Cerrado and Amazon) and could not be set to occur precisely in the Amazon were not included in the list.

\section{RESULTS}

A list of 469 species is presented (Table 1), including 52 new occurrences for the Brazilian Amazon (indicated by "NEW"). Fourteen species appear as new records for the municipalities and their respective states (indicated by "AMZ").

We recorded 102 genera, of which 34 were monospecific. The genera Trichromia Hübner, [1819] had the highest number of species (58), followed by Zatrephes Hübner, [1819] (36), Amaxia Walker, 1855 (24), and Leucanopsis Rego Barros, 1956 (23). The species with the highest number of records were Psychophasma erosa (Herrich-Schäffer, [1858]) and Azatrephes discalis (Walker, 1856) (both with 27), followed by Ammalo helops (Cramer, [1776]) and Viviennea moma (Schaus, 1905) (both with 18), and Euplesia sphingidea (Perty, [1833]) (with 17). A total of 154 species (32.8\%) occurred in only one locality (Table 1 ).

All nine Brazilian states that comprise the biome showed occurrence of Phaegopterina. In total, 69 Amazonian municipalities had records of phaegopterine species. Fonte Boa (AM), Belém (PA), and Cacaulândia (RO) were the municipalities with the highest number of species, with 240,184 , and 126 , respectively.

\section{DISCUSSION}

The cities with the largest number of species are widely sampled. Fonte Boa and Belém tiger moths were recorded by several naturalists who sampled in the Amazon in the 19th and 20th centuries. In the case of Cacaulândia, the species were collected by Vitor Becker in the 1990s. The tiger moth richness of these locations is among the largest recorded in Brazilian sites. Only Itatiaia (382), Petrópolis (355), Angra dos Reis (331) (Ferro and Melo 2011), Boracéia (237) (Ferro and Diniz 2007), Joinville (162), and Seara (129) (Ferro et al. 2012), all located in the Atlantic Forest, had more species recorded than Fonte Boa, Belém, and Cacaulândia. However, the locations of the Atlantic Forest encompass the entire subfamily, while the municipalities of our study encompass only the subtribe Phaegopterina.

The number of phaegopterine species recorded for the Brazilian Amazon is almost twice that found in the Brazilian Cerrado (Ferro et al. 2010) and corresponds to $27.3 \%$ of Neotropical phaegopterine species (Vincent and Laguerre 2014). Ferro and Diniz (2010) recorded 753 species of Arctiinae in the Amazon. Due to the new records obtained in our study (52), there was an increase of approximately $7 \%$ in richness for the biome, generating a total of 805 tiger moth Amazon species. This makes the Amazon the second largest Brazilian biome in arctiid richness, after the Atlantic Forest (1,191 species; Ferro and Melo 2011). However, we believe that the richness of arctiid moths in the Amazon is underestimated. As argued by Santos et al. (2008) regarding butterflies, the northern region of Brazil is very poorly sampled. The large geographical area, difficult access, high costs of expeditions, and distance from urban and research centers probably contribute to the lack of studies in the Amazon.

\section{ACKNOWLEDGEMENTS}

We are indebted to the museum curators for allowing VGF's access to the collections under their care and for logistical support. The Dr. Benoit Vincent for help in identifying some species and articles sent to us. This publication is part of the RedeLep "Rede Nacional de Pesquisa e Conservação de Lepidópteros", SISBIOTABrasil, CNPq (563332/2010-7).

\section{LITERATURE CITED}

Ab'Saber, A.N. 1977. Os domínios morfoclimáticos na América do Sul. Primeira aproximação. Geomorfologia 52: 1-22.

Bryk, F. 1953. Lepidoptera aus dem amazonasgebiete und aus Peru gesammelt von Dr. Douglas Melin und Dr. Abraham Roman. Arkiv för Zoologi 5: 1-268.

Butler, A.G. 1876. On the subfamilies Antichlorinae and Charideinae of the lepidopterous families Zygaenidae and Arctiidae. Journal of the Linnean Society (Zoology) 12: 408-433.

Butler, A.G. 1877. Illustrations of typical specimens of Lepidoptera Heterocera in the British Museum. Part 1. London: Taylor and Francis. 62 pp. doi: 10.5962/bhl.title.31402

Butler, A.G. 1878. On the Lepidoptera of the Amazons, collected by Dr James W. H. Trail during the years 1873 to 1875 . Transactions of the Entomological Society of London 1878: 39-84.

Coenen, F. and C. Gibeaux. 2014a. Toulgoetarctia haematora n. gen., n. sp., de la Guyane (Lepidoptera Erebidae Arctiinae Arctiini) (4ème note). Lambillionea 114(1): 27-36.

Coenen, F. and C. Gibeaux. 2014b. Description de Chrysomallos, genre nouveau dans la sous-famille des Arctiinae (Lepidoptera, Erebidae, Arctiinae, Phaegopterini) (5ème note). Lambillionea 114(2): 132-138.

Costa Lima, A.M. da. 1950. Insetos do Brasil. Lepidópteros. $6^{\circ}$ tomo, $2^{\mathrm{a}}$ parte. Rio de Janeiro: Escola Nacional de Agronomia. 420 pp.

D’Almeida, R.F. 1968. Algunas considerações sobre Arctiidae brasileiros, com as descrições de duas espécies novas (Lepidoptera-Heterocera). Revista de la Sociedad Entomologica Argentina 30: 3-7.

Dognin, P. 1921. Hétérocères nouveaux de l'Amérique du Sud Fascicule XIX. Rennes: Imprimerie Oberthür. 19 pp. doi: 10.5962/ bhl.title.59881

Dognin, P. 1923a. Hétérocères nouveaux de l'Amérique du Sud Fascicule XXI. Rennes: Imprimerie Oberthür. 38 pp. doi: 10.5962/bhl.title.59881

Dognin, P. 1923b. Hétérocères nouveaux de l'Amérique du Sud Fascicule XXII. Rennes: Imprimerie Oberthür. 25 pp. doi: 
10.5962/bhl.title.59881

Dognin, P. 1923c. Hétérocères nouveaux de l'Amérique du Sud Fascicule XXIII. Rennes: Imprimerie Oberthür. 34 pp. doi: 10.5962/bhl.title.59881

Dognin, P. 1924. Hétérocères nouveaux de l'Amérique du Sud. Fascicule XXV. Rennes: Imprimerie Oberthür. 34 pp.

Druce, H. 1890. Descriptions of new species of Lepidoptera Heterocera from Central and South America. Proceedings of the Zoological Society of London 34: 493-520.

Druce, H. 1899. Descriptions of some new species of Lepidoptera Heterocera from Tropical America, Africa, India, and the Eastern islands. The Annals and Magazine of Natural History [seventh series] 3: 465-474.

Emery, E. de O. 2006. O gênero neotropical Cresera (Lepidoptera, Arctiinae, Phaegopterini). Dissertação (Mestrado em Biologia Animal). Universidade de Brasília, Instituto de Ciências Biológicas, Brasília. 135 pp.

Fearnside, P.M. 2003. The Amazon rainforest in global change. Manaus: INPA. 134 pp.

Felder, R. and A.F. Rogenhofer. 1874-1875. in Felder, C., R. Felder and A.F. Rogenhofer. Reise der österreichischen fregatte novara um die Erde (Zool.), 2 (Abt. 2): pl 75-120 (1874); Erklärung to pls 75-107 (1875). Vienna.

Ferreira, L.V., E. Venticinque and S. Almeida. 2005. O desmatamento na Amazônia e a importância das áreas protegidas. Estudos avançados 19 (53): 157-166. doi: 10.1590/S0103-40142005000 100010

Ferro, V.G. and I.R. Diniz. 2007. Arctiidae (Insecta: Lepidoptera) da Estação Biológica de Boracéia (Salesópolis, São Paulo, Brasil). Biota Neotropica 7 (3): 331-338.

Ferro, V.G. and I.R. Diniz. 2010. Riqueza e composição das mariposas Arctiidae (Lepidoptera) no Cerrado; pp. 255-313, in: I.R. Diniz, J. Marinho-Filho, R.B. Machado and R. Cavalcanti (eds.). Cerrado: conhecimento quantitativo como subsídio para as ações de conservação. Brasília: Editora Thesaurus.

Ferro, V.G. and A.S. Melo. 2011. Diversity of tiger moths in a Neotropical hotspot: determinants of species composition and identification of biogeographic units. Journal of Insect Conservation 15: 643-651. doi: 10.1007/s10841-010-9363-6

Ferro, V.G., A.S. Melo and I.R. Diniz. 2010. Richness of tiger moths (Lepidoptera: Arctiidae) in the Brazilian Cerrado: how much do we know? Zoologia 27: 725-731.

Ferro, V.G., I.M.H Resende and M. Duarte. 2012. Arctiinae (Lepidoptera: Erebidae) do estado de Santa Catarina, Brasil. Biota Neotropica 12(4): 166-180.

Gaede, M. 1923. Alte und neue Arctiinae des Berliner Zoologischen Museums. Entomologische Rundschau 40 (7): 27-28.

Gaede, M. 1928. Alte und neue Arctiinae des Berliner Zoologischen Museums. Entomologische Rundschau 45 (9): 33-34.

Hampson, G.F. 1898. Catalogue of the Lepidoptera Phalaenae in the British Museum. Volume 1. Catalogue of the Syntomidae in the collection of the British Museum. London: Taylor and Francis. $559 \mathrm{pp}$.

Hampson, G.F. 1901. Catalogue of the Lepidoptera Phalaenae in the British Museum. Volume 3. Catalogue of the Arctiadae (Arctianae) and Agaristidae in the collection of the British Museum. London: Taylor and Francis. 690 pp.

Hampson, G.F. 1920. Catalogue of the Lepidoptera Phalaenae in the British Museum. Supplement. Volume II.: Catalogue of the Lithosiadae (Arctianae) and Phalaenoididae in the collection of the British Museum. London: Taylor and Francis. 619 pp.

Hawes, J., C. da S. Motta, W.L. Overal, J. Barlow, T.A. Gardner and C.A. Peres. 2009. Diversity and composition of Amazonian moths in primary, secondary and plantation forest. Journal of Tropical Ecology 25(3): 281-300. doi: 10.1017/S0266467409006038
Heppner, J.B. 1991. Faunal regions and the diversity of Lepidoptera. Tropical Lepidoptera 2(suppl. 1): 1-85.

IBGE (Instituto Brasileiro de Geografia e Estatística). 2014. IBGE Cidades@. Accessed at http://www.cidades.ibge.gov.br/xtras/ home.php, 7 June 2014.

Jacobson, N.L. and S.J. Weller. 2002. A cladistic study of the Arctiidae (Lepidoptera) by using characters of immatures and adults. Lanham: Thomas Say Publications in Entomology (Monograph)/ Entomological Society of America. 98 pp.

Kirby, W.F. 1892. A synonymic catalogue of Lepidoptera Heterocera (Moths). Vol. I. Sphinges and Bombyces. Taylor and Francis: London. 951 pp.

Kitching, I.J. and J.E. Rawlins. 1999. The Noctuoidea; pp. 355-401, in: N.P. Kristensen (ed.). Lepidoptera, moths and butterflies. Volume 1: evolution, systematics, and biogeography. in: $\mathrm{M}$. Fischer (ed.). Handbook of zoology. Volume IV Arthropoda: insecta. Berlin: de Gruyter.

Perty, M. 1833. Insecta brasiliensia; pp. 125-224, in: J.B. de Spix and K.F. Ph. de Martius (eds.). Delectus animalium articulatorum quae in itinere per Brasiliam annis MDCCCXVII-MDCCCXXjussu et auspiciis Maximiliani Josephi I. Bavariae Regis Augustissimi. Munich: Frid. Fleischer.

Piñas Rubio, F., S. Rab-Green, G. Onore and I. Manzano. 2000. Mariposas del Ecuador Vol. 20. Familia: Arctiidae Subfamilias: Arctiinae y Pericopinae. Quito: Pontificia Universidad Catolica del Ecuador. 126 pp.

Rego Barros, A.R. do. 1946. Contribuição ao estudo da família Arctiidae - II Estudo do gênero Psychophasma (Lepidoptera Heterocera). Boletim do Museu Nacional 63: 1-8.

Rego Barros, A.R. do. 1955. Fauna do Distrito Federal. XXXIII: Sôbre o gênero "Ammalo" Walker, 1855 (Lepidoptera, Heterocera). Revista Brasileira de Biologia 15: 223-237.

Rego Barros, A.R. do. 1956. XL - Fauna do Distrito Federal. Sôbre Paraeuchaetes Grote, 1865 com descrição de espécies novas (Lepidoptera-Heterocera). Revista Brasileira de Entomologia 6: 65-90.

Rego Barros, A.R. do. 1958. Contribuição ao estudo da família Arctiidae. VII - Rediagnose do gênero Cresera Schaus, 1894 com descrição de nova espécie (Lepidoptera-Heterocera). Anais da Acadêmia Brasileira de Ciências 30: 363-390.

Rego Barros, A.R. do. 1968. Contribuição ao estudo da família Arctiidae Sobre três espécies do gênero Automolis Hampson, 1901. Boletim do Museu Nacional 258: 1-16.

Rego Barros, A.R. do. 1971. Contribuição ao estudo da família Arctiidae. II - Revisão do gênero Elysius Walker, 1855 com descrição de novas espécies e revalidação do gênero Onythes Walker, 1855 (Lepidoptera-Heterocera). Arquivos do Museu Nacional 54: 121-127.

Reich, P. 1933a. Die Neue südamerikanische Arctiiden (Phaegopterini). Entomologische Rundschau 50: 258-260.

Reich, P. 1933b. Die Neue südamerikanische Arctiiden (Phaegopterini). Entomologische Rundschau 50: 280-282.

Reich, P. 1934. Neue südamerikanische Arctiidae. Internationale Entomologische Zeitschrift 28: 389-391.

Reich, P. 1937a. Die Bärenspinner von Südbrasilien. Faunistische Mitteilungen aus meiner Sammlung. Entomologische Rundschau 54: 563-564.

Reich, P. 1937b. Die Bärenspinner von Südbrasilien. Faunistische Mitteilungen aus meiner Sammlung. Entomologische Rundschau 55: 69-73.

Rothschild, L.W. 1909a. Description of some new South American Arctiadae, with notes. Novitates Zoologicae 16: 21-52.

Rothschild, L.W. 1909b. Description of some new South American Arctiadae, with notes. Novitates Zoologicae 16: 268-299.

Rothschild, L.W. 1909c. Descriptions of new South-American Arcti- 
anae. The Annals and Magazine of Natural History [8th series] 4: 205-229.

Rothschild, L.W. 1910. Catalogue of the Arctianae in the Tring Museum, with notes and descriptions of new species. Novitates Zoologicae 18: 1-85.

Rothschild, L.W. 1911. New Syntomidae and Arctianae. Novitates Zoologicae 18: 154-158.

Rothschild, L.W. 1913. New Lithosianae. Novitates Zoologicae 20: 192-226.

Rothschild, L.W. 1917. Some new moths of the families Arctiidae and Eupterotidae. Novitates Zoologicae 24: 475-492.

Rothschild, L.W. 1922. A preliminary list of the Arctiinae of Pará, Brazil, and a few from other localities. The Annals and Magazine of Natural History [9th series] 9: 457-494.

Rothschild, L.W. 1933. New species and subspecies of Arctiinae. The Annals and Magazine of Natural History [10th series] 11: 167-194.

Rothschild, L.W. 1935. Some new Arctiinae. Novitates Zoologicae 39: 239-250.

Santos, E.C., O.H.H. Mielke and M.M. Casagrande. 2008. Inventários de borboletas no Brasil: estado da arte e modelo de áreas prioritárias para pesquisa com vistas à conservação. Natureza e Conservação 6: 68-90.

Santos, G.P. 2006. Lepidópteros associados a plantios de eucalipto na região Amazônica do Brasil: diversidade alfa e beta e impacto dos fatores ambientais. Tese (Doutorado em Entomologia). Universidade Federal de Viçosa, Programa de Pós-graduação em Entomologia, Viçosa, MG. 95 pp.

Schaus, W. 1933. New species of Heterocera in the National Museum. Annals and Magazine of Natural History [10th series] 11: 566-587.

Seitz, A. 1919-1925. 4. Familie: Arctiidae, Bärenspinner; p. 231-497, in: A. Seitz (ed.). Die Gross-Schmetterlinge der Erde. II. Abteilung: Die Gross-Schmetterlinge des Amerikanischen Faunengebietes. 6. Band. Die Amerikanischen Spinner und Schwärmer. Stuttgart: Alfred Kernen.

Teston, J.A., A.C.W. da Conceição, D.M.P. Valente and M.P. de Freitas. In review. First record of Araeomolis propinqua Toulgoët, 1998 (Lepidoptera: Erebidae: Arctiinae) from Brazil. Check List.

Teston, J.A. and D. do C.V. Correa. 2015. The Arctiini (Lepidoptera, Erebidae, Arctiinae) fauna of the Serra do Pardo National Park, Pará, Brazil. Check List 11(2): 1613. doi: 10.15560/11.2.1613

Teston, J.A. and M.C. Delfina. 2010. Diversidade de Arctiinae (Lepidoptera, Arctiidae) em área alterada em Altamira, Amazônia Oriental, Pará, Brasil. Acta Amazonica 40(2): 387-396. doi: 10.1590/S0044-59672010000200017

Teston, J.A. and M.P. de Freitas. 2015. First record of two species of Gorgonidia Dyar, 1898 (Lepidoptera: Erebidae: Arctiinae) from Brazil. Check List 11(3) 1651. doi: 10.15560/11.3.1651

Travassos, L. 1943. Contribuição ao conhecimento dos "Arctiidae". I. (Lepidoptera, Heterocera). Revista Brasileira de Biologia 3: 453-472.

Travassos, L. 1944a. Contribuição ao conhecimento dos "Arctiidae". II. (Lepidoptera, Heterocera). Revista Brasileira de Biologia 4: $1-12$.

Travassos, L. 1944b. Contribuição ao conhecimento dos "Arctiidae". III. (Lepidoptera, Heterocera). Revista Brasileira de Biologia 4: 151-155.

Travassos, L. 1944c. Contribuição ao conhecimento dos "Arctiidae". IV. (Lepidoptera, Heterocera). Revista Brasileira de Biologia 4: 297-312.

Travassos, L. 1944d. Contribuição ao conhecimento dos "Arctiidae". V. (Lepidoptera, Heterocera). Revista Brasileira de Biologia 4: 439-451.

Travassos, L. 1945a. Contribuição ao conhecimento dos "Arctiidae". VIII. (Lepidoptera, Heterocera). Revista Brasileira de Biologia 5:
197-204.

Travassos, L. 1945b. Contribuição ao conhecimento dos "Arctiidae". X. (Lepidoptera, Heterocera). Revista Brasileira de Biologia 5: 509-523.

Travassos, L. 1949. Contribuição ao conhecimento dos "Arctiidae". XVIII. (Lepidoptera, Heterocera). Revista Brasileira de Biologia 9: 179-186.

Travassos, L. 1950. Contribuição ao conhecimento dos "Arctiidae". XXI. Sôbre as espécies de coloração semelhante a "Idalus admirabilis" (Cramer, 1777). Revista Brasileira de Biologia 10: 217-240.

Travassos, L. 1951a. Contribuição ao conhecimento dos "Arctiidae". XXIII. Gênero "Purius" Walker, 1855. Revista Brasileira de Biologia 11: 43-47.

Travassos, L. 1951b. Contribuição ao conhecimento dos "Arctiidae". XXV. Sôbre o gênero "Arctiarpia" n. g. Revista Brasileira de Biologia 11: 249-253.

Travassos, L. 1951c. Contribuição ao conhecimento dos "Arctiidae". XXVI. Sôbre o gênero "Xanthoarctia" n. g. Revista Brasileira de Biologia 11: 393-398.

Travassos, L. 1952. Contribuição ao conhecimento dos "Arctiidae". XXVII. Redescrição de três espécies do gênero "Rhipha" incluidas por Seitz no gênero "Idalus" (Lepidoptera, Heterocera). Revista Brasileira de Biologia 12: 49-57.

Travassos, L. 1956. Contribuição ao conhecimento dos "Arctiidae". XXXVI. Sôbre o gênero "Graphaea" Schaus, 1894. Revista Brasileira de Biologia 16: 443-452.

Travassos, L. 1957. Contribuição ao conhecimento dos "Arctiidae". XXXVII. Gênero "Castrica" Schaus, 1896. Revista Brasileira de Biologia 17: 227-234.

Travassos, L. 1959. Contribuição ao conhecimento dos "Arctiidae". XL. Gênero "Amaxia" Waker, 1855 (Lepidoptera, Heterocera). Revista Brasileira de Biologia 19: 307-318.

Travassos, L. 1963. Contribuição ao conhecimento dos "Arctiidae". XL. O Problema Halisidota (Lepidoptera). Memórias do Instituto Oswaldo Cruz 61: 471-490.

Travassos, L. 1964. Contribuição ao conhecimento dos Arctiidae. Gênero Robinsonia Grote, 1865 (Lepidoptera, Heterocera). Anais da Acadêmia Brasileira de Ciências 36: 515-537.

Vieira, I.C.G., P.M. Toledo, J.M.C. Silva and H. Higuchi. 2008. Deforestation and threats to the biodiversity of Amazonia. Brazilian Journal of Biology 68(4, Suppl.): 949-956.

Vincent, B. and M. Laguerre. 2014. Catalogue of the Neotropical Arctiini Leach, [1815] (except Ctenuchina Kirby, 1837 and Euchromiina Butler, 1876) (Insecta, Lepidoptera, Erebidae, Arctiinae). Zoosystema 36(2): 137-533. doi: 10.5252/z2014n2a1

Walker, F. 1854a. List of the specimens of lepidopterous insects in the collection of the British Museum. Part I.- Lepidoptera Heterocera. London: Edward Newman. pp. 1-278.

Walker, F. 1854b. List of the specimens of lepidopterous insects in the collection of the British Museum. Part II.- Lepidoptera Heterocera. London: Edward Newman. pp. 279-581.

Walker, F. 1855. List of the specimens of lepidopterous insects in the collection of the British Museum. Part III.- Lepidoptera Heterocera. London: Edward Newman. pp. 582-775.

Walker, F. 1856. List of the specimens of lepidopterous insects in the collection of the British Museum. Part VII.- Lepidoptera Heterocera. London: Edward Newman. pp. 1509-1808.

Walker, F. 1865. List of the specimens of lepidopterous insects in the collection of the British Museum. Part XXXI. Supplement. London: Edward Newman. pp. 1-322. doi: 10.5962/bhl. title. 58221

Watson, A. 1971. An illustrated catalog of the Neotropic Arctiinae types in the United States National Museum (Lepidoptera: Arctiidae) part I. Smithsonian Contributions to Zoology 50: $1-361$.

Watson, A. 1973. An illustrated catalog of the Neotropic Arctiinae 
types in the United States National Museum (Lepidoptera: Arctiidae) part II. Smithsonian Contributions to Zoology 128: $1-160$

Watson, A. 1975. A reclassification of the Arctiidae and Ctenuchidae formerly placed in the thyretid genus Automolis Hübner (Lepidoptera). With notes on warning coloration and sound. Bulletin of the British Museum Natural History (Entomology) 25: 1-104. [Supplement].

Watson, A.1980. A revision of the Halysidota tessellaris species-group (Halysidota sensu stricto) (Lepidoptera: Arctiidae). Bulletin of the British Museum Natural History (Entomology) 40: 1-65.

Watson, A., D.S. Fletcher and I.W.B. Nye. 1995. Noctuoidea: Arctiidae, Cocytiidae, Ctenuchidae, Dilobidae, Dioptidae, Lymantriidae, Notodontidae, Strepsimanidae, Thaumetopoeidae \& Thyretidae.
Volume 2; 228 pp., in: I.W.B. Nye (ed.). The generic names of moths of the world. Reprinted. London: The Natural History Museum.

Zahiri, R., J.D. Holloway, I.J. Kitching, J.D. Lafontaine, M. Mutanen and N. Wahlberg. 2012. Molecular phylogenetics of Erebidae (Lepidoptera, Noctuiodea). Systematic Entomology 37: 102-124. doi: 10.1111/j.1365-3113.2011.00607.x

Author contributions: JT and VF collected the data and wrote the text. JT organized the species list.

Received: 11 March 2015

Accepted: 4 March 2016

Academic editor: Reza Zahiri

Table 1. Phaegopterina (Erebidae, Arctiinae, Arctiini) species of the Brazilian Amazon. The record column shows the Brazilian state in abbreviated form followed by municipality. The name of the locality is enclosed in braces and the author of the first record is in parentheses. * New record.

\begin{tabular}{|c|c|}
\hline Species & Record \\
\hline Agaraea klagesi Rothschild, 1909 & AM, Fonte Boa (Rothschild 1909b) \\
\hline Agaraea minuta (Schaus, 1892) & $\begin{array}{l}\text { AM, Fonte Boa*; PA, Altamira \{Monte Santo\} (Teston and Delfina 2010), Belém*, Capitão Poço*; RO, Ariquemes*, } \\
\text { Cacaulândia*, Porto Velho* }\end{array}$ \\
\hline Agaraea nigrotuberculata (Bryk, 1953) & AM, Rio Purús (Bryk 1953) \\
\hline Agaraea semivitrea (Rothschild, 1909) & $\begin{array}{l}\text { AM, Codajás (Rothschild 1910), Manaus (Bryk 1953), São Paulo de Olivença*; MA, Açailândia*; PA, Almeirim \{Jari\} } \\
\text { (Hawes et al. 2009), [Belém] (Rothschild 1922), Novo Progresso*, Santarém* }\end{array}$ \\
\hline Amaxia apyga Hampson, 1901 & $\begin{array}{l}\text { AM, Benjamin Constant*, Fonte Boa (Rothschild 1910), Manaus \{Uypiranga\} (Reich 1937a), São Paulo de Olivença } \\
\text { (Hampson 1901); AP, Amapari*, Serra do Navio*; RO, Cacaulândia*, Candeias do Jamari, Porto Velho* }\end{array}$ \\
\hline Amaxia beata (Dognin, 1909) & $\begin{array}{l}\text { AM, Benjamin Constant*, Fonte Boa (Rothschild 1910), Manaus*, São Paulo de Olivença*; AP, Serra do Navio*; MA, } \\
\text { Açailândia*; PA, São Félix do Xingu \{Serra do Pardo National Park\} (Teston and Correa 2015); RO, Cacaulândia* }\end{array}$ \\
\hline Amaxia bella (Schaus, 1905) & $\begin{array}{l}\text { AM, Fonte Boa (Rothschild 1910), Manaus*; AP, Serra do Navio*; PA, [Belém] (Rothschild 1922), Capitão Poço*, } \\
\text { Marabá*; RO, Cacaulândia*, Jaru*, Porto Velho*; RR, Alto Alegre** }\end{array}$ \\
\hline Amaxia carinosa Schaus, 1920 & PA, Almeirim \{Jari\} (Hawes et al. 2009) \\
\hline Amaxia chaon (Druce, 1883) & $\begin{array}{l}\text { AM, Benjamin Constant*, Fonte Boa (Rothschild 1910), Manaus (Bryk 1953), Moura*, São Gabriel da Cachoeira } \\
\text { \{Querari\}*; AP, Serra do Navio*; MT, Sinop*; PA, [Belém] (Rothschild 1910), São Félix do Xingu \{Serra do Pardo } \\
\text { National Park\} (Teston and Correa 2015); RR, Alto Alegre* }\end{array}$ \\
\hline Amaxia consistens Schaus, 1905 & $\begin{array}{l}\text { AM, Atalaia do Norte \{Santo Antonio do Javari\} (Rothschild 1910), Benjamin Constant*, Borba*, Fonte } \\
\text { Boa (Rothschild 1922); MA, Açailândia*; PA, Almeirim \{Jari\} (Hawes et al. 2009), [Belém] (Rothschild 1922), } \\
\text { Parauapebas*, São Félix do Xingu \{Serra do Pardo National Park\} (Teston and Correa 2015); RO, Cacaulândia*, } \\
\text { Porto Velho** }\end{array}$ \\
\hline Amaxia disconsistens Dognin, 1923 & AM, São Paulo de Olivença (Dognin 1923b) \\
\hline Amaxia egaensis (Seitz, 1921) & $\begin{array}{l}\text { AM, Benjamin Constant*, Borba*, Fonte Boa*, São Paulo de Olivença*, Tefé (Seitz 1921); MA, Açailândia*; RO, } \\
\text { Candeias do Jamari* }\end{array}$ \\
\hline Amaxia erythrophleps Hampson, 1901 & $\begin{array}{l}\text { AM, Fonte Boa (Rothschild 1910), São Gabriel da Cachoeira \{Querari\}*; AP, Serra do Navio*; MA, Açailândia*; PA, } \\
\text { Altamira \{Serra do Pardo National Park\} (Teston and Correa 2015), Marabá*; São Félix do Xingu \{Serra do Pardo } \\
\text { National Park\} (Teston and Correa 2015); RO, Cacaulândia*, Porto Velho* }\end{array}$ \\
\hline Amaxia flavicollis (Rothschild, 1909) & $\begin{array}{l}\text { AM, Fonte Boa (Rothschild 1909a); PA, [Belém] (Rothschild 1922), São Félix do Xingu \{Serra do Pardo National } \\
\text { Park\} (Teston and Correa 2015) }\end{array}$ \\
\hline Amaxia gnosia (Schaus, 1905) & $\begin{array}{l}\text { AM, Atalaia do Norte \{Santo Antonio do Javari\} (Rothschild 1910), Fonte Boa (Rothschild 1910); RO, Porto Velho } \\
\text { (Rothschild 1910) }\end{array}$ \\
\hline Amaxia kennedyi (Rothschild, 1909) AMz & MA, Açailândia* \\
\hline Amaxia klagesi (Rothschild, 1909) NEW & AM, Manaus*; RO, Porto Velho* \\
\hline Amaxia laurentia (Schaus, 1905) NEW & MT, Sinop* \\
\hline Amaxia lepida (Schaus, 1912) & PA, Altamira \{Monte Santo\} (Teston and Delfina 2010) \\
\hline Amaxia manora (Druce, 1906) & AM, Fonte Boa (Rothschild 1910) \\
\hline Amaxia ockendeni (Rothschild, 1909) & AM, Fonte Boa (Rothschild 1909a); RO, Porto Velho* \\
\hline Amaxia pandama (Druce, 1893) & $\begin{array}{l}\text { AM, Benjamin Constant*, Fonte Boa (Rothschild 1910); MT, Aripuanã*; PA, [Belém] (Rothschild 1922), Marabá*, } \\
\text { São Félix do Xingu \{Serra do Pardo National Park\} (Teston and Correa 2015); RO, Cacaulândia** }\end{array}$ \\
\hline Amaxia pardalis Walker, 1855 & $\begin{array}{l}\text { AM, Borba \{Lago Acará\} (Travassos 1959), Manaus (Rothschild 1909a), Rio Purús (Bryk 1953), Tefé (Walker 1855); } \\
\text { PA, Almeirim \{Jari\} (Hawes et al. 2009) }\end{array}$ \\
\hline Amaxia pseudodyuna Rothschild, 1922 & PA, [Belém] (Rothschild 1922) \\
\hline Amaxia pyga Schaus, 1892 & AM, Rio Amazonas (Rothschild 1910); PA, Oriximiná* \\
\hline Amaxia reticulata (Rothschild, 1909) & $\begin{array}{l}\text { AM, Fonte Boa (Rothschild 1909a); AP, Serra do Navio*; MA, Açailândia*; PA, Marabá*, São Félix do Xingu \{Serra do } \\
\text { Pardo National Park\} (Teston and Correa 2015); RO, Porto Velho* }\end{array}$ \\
\hline Amaxia rufobasalis Rothschild, 1909 & AM, Fonte Boa (Rothschild 1909a); RO, Cacaulândia*, Porto Velho* \\
\hline Amaxia theon Druce, 1900a & PA, Almeirim \{Jari\} (Hawes et al. 2009); RO, Porto Velho* \\
\hline Ammalo ammaloides (Rothschild, 1909) & AM, Fonte Boa (Rothschild 1909c) \\
\hline
\end{tabular}


Table 1. Continued.

\begin{tabular}{l}
\hline Species \\
\hline Ammalo klagesi Rothschild, 1909 \\
Ammalo helops (Cramer, [1776])
\end{tabular}

Ammalo violitincta Rothschild, 1922 Amphelarctia priscilla (Schaus, 1911) Aphyle affinis Rothschild, 1909 Aphyle cuneata Hampson, 1905

Aphyle margaritaceus Walker, 1855 Apiconoma opposita (Walker, 1854)

Apyre separata Walker, 1854

Araeomolis albipicta (Dognin, 1909) Araeomolis irregularis (Rothschild, 1909) Araeomolis irrupta (Schaus, 1905) Araeomolis persimilis Rothschild, 1909

Araeomolis propinqua Toulgoët, 1998 Araeomolis rhodographa rhodographa Hampson, 1901

Araeomolis rubens (Schaus, 1905) Arctagyrta nana (Walker, 1856) Arctiarpia melanopasta (Dognin, 1907)

Astralarctia canalis (Schaus, 1921) NEW Astralarctia pulverosa (Schaus, 1905)

Azatrephes argyrotis orientalis Rothschild, 1922 Azatrephes discalis (Walker, 1856)

Azatrephes fuliginosa Rothschild, 1909 Baritius acuminata (Walker, 1856) NEW Baritius affinis Rothschild, 1910 Baritius brunnea Hampson, 1901 Baritius cyclozonata Hampson, 1901

Baritius eleuthera (Stoll, [1781])

Baritius eleutheroides Rothschild, 1909

Baritius flavescens Rothschild, 1909 Baritius sannionis (Rothschild, 1909)

Bertholdia detracta Seitz, 1921

Biturix hoffmannsi Rothschild, 1909 Biturix intactus Walker, 1855 Biturix obscura (Hampson, 1898)

Biturix pellucida (Sepp, [1852])
Record

AM, Fonte Boa (Rothschild 1909c)

AM, Benjamin Constant (Rego Barros 1955), Borba*, Codajás (Rothschild 1910), Fonte Boa (Rothschild 1910), Manaus (Rego Barros 1955), Moura \{Rio Negro\} (Rego Barros 1955), Rio Solimões (Rego Barros 1955), São Paulo de Olivença (Rego Barros 1955); AP, Serra do Navio*; MT, Sinop*; PA, Alenquér*, Almeirim \{Jari\} (Hawes et al. 2009), [Belém] (Rothschild 1922), Óbidos (Rego Barros 1955), São Félix do Xingu \{Serra do Pardo National Park\} (Teston and Correa 2015); RO, Cacaulândia*, Porto Velho \{Calama\} (Rothschild 1910); RR, Alto Alegre*

PA, [Belém] (Rothschild 1922)

AM, Codajás (Watson 1975), Fonte Boa (Watson 1975)

AM, Fonte Boa (Rothschild 1909a), Tefé (Rothschild 1910)

AM, Benjamin Constant*, Fonte Boa (Rothschild 1910), Moura*, São Paulo de Olivença*; MT, Sinop*; PA, Almeirim \{Jari\} (Hawes et al. 2009), Belém*; RO, Porto Velho*

AM, Fonte Boa*, Tefé (Seitz 1921); PA, [Belém] (Walker 1855), Capitão Poço*, Viseu*; RO, Porto Velho*

AM, Fonte Boa (Rothschild 1910), Manaus (Travassos 1944d), Moura*, Tefé (Travassos 1944d); AP, Serra do Navio*; MA, Açailândia*; PA, [Belém] (Rothschild 1922), Marabá*; RO, Cacaulândia*, Candeias do Jamari*, Porto Velho*

AM, Benjamin Constant*, Fonte Boa*, Manaus*, Moura*, São Gabriel da Cachoeira*, Tefé (Walker 1854b); AP, Serra do Navio*; MA, Açailândia*; PA, Altamira \{Serra do Pardo National Park\} (Teston and Correa 2015), [Belém] (Rothschild 1922), Marabá*, São Félix do Xingu \{Serra do Pardo National Park\} (Teston and Correa 2015); RO, Cacaulândia*, Candeias do Jamari*, Porto Velho*; RR, Alto Alegre*

AM, Fonte Boa (Rothschild 1910), Manaus*, Moura*; AP, Serra do Navio*; MA, Açailândia*; RO, Porto Velho* AM, Fonte Boa (Rothschild 1909a)

AM, Manaus*; AP, Serra do Navio*; MA, Açailândia*; PA, Almeirim \{Jari\} (Hawes et al. 2009)

AM, Atalaia do Norte \{Santo Antonio do Javari\} (Rothschild 1910), Codajás (Rothschild 1910), Fonte Boa (Rothschild 1909b), Humaitá (Rothschild 1909b), Rio Purús (Bryk 1953); RO, Porto Velho \{Aliança\} (Rothschild 1909b)

AM, Manaus*; PA, Itaituba \{Amazônia National Park\} (Teston et al. 2016)

AM, Atalaia do Norte \{Santo Antonio do Javari\} (Rothschild 1910), Barcelos \{Tomar\} (Hampson 1901), Benjamin Constant*, Borba*, Codajás \{Rio Negro\} (Rothschild 1910), Fonte Boa (Rothschild 1910), Manicoré*, São Paulo de Olivença*, Tefé (Rothschild 1910); AP, Serra do Navio*; MT, Aripuanã*; PA, [Belém] (Rothschild 1922), São Félix do Xingu \{Serra do Pardo National Park\} (Teston and Correa 2015); RO, Cacaulândia*

AM, Fonte Boa (Rothschild 1910), Manaus*; MA, Açailândia*; MT, Sinop*; PA, Almeirim \{Jari\} (Hawes et al. 2009) AM, Rio Purús (Bryk 1953), Vale do [Rio] Amazonas (Walker 1856)

AM, Benjamin Constant*, Fonte Boa (Rothschild 1922), Humaitá (Rothschild 1910), Manaus (Rothschild 1910), São Paulo de Olivença*; MT, Aripuanã*; PA, [Belém] (Rothschild 1922), São Félix do Xingu \{Serra do Pardo National Park\} (Teston and Correa 2015) ; RO, Cacaulândia*, Jaru*, Porto Velho*

MA, Açailândia*; RO, Porto Velho*

AM, Benjamin Constant* Fonte Boa (Rothschild 1910), Manaus \{Uypiranga\} (Reich 1933b), São Paulo de Olivença*; AP, Serra do Navio*; MT, Aripuanã*, Sinop*; PA, Marabá*, São Félix do Xingu \{Serra do Pardo National Park\} (Teston and Correa 2015); RO, Cacaulândia*, Porto Velho*

PA, [Belém] (Rothschild 1922)

AC, Santa Rosa do Purus*; AM, Benjamin Constant*, Borba*, Codajás (Rothschild 1910), Fonte Boa (Rothschild 1910), [Jutaí] \{Rio Jutahi\} (Butler 1877), Manaus*, Moura*, São Paulo de Olivença*, Tefé (Rothschild 1910); AP, Serra do Navio*; MT, Sinop*; PA, Almeirim \{Jari\} (Hawes et al. 2009), Altamira \{Monte Santo\} (Teston and Delfina 2010) and \{Serra do Pardo National Park\} (Teston and Correa 2015), [Belém] (Rothschild 1922), Benevides*, Gurupá*, Novo Progresso \{Cachimbo\}*, Ourém*, São Félix do Xingu \{Serra do Pardo National Park\} (Teston and Correa 2015); RO, Ariquemes*, Cacaulândia*, Candeias do Jamari*, Jaru*, Porto Velho (Rothschild 1910) AM, Fonte Boa (Rothschild 1909a); RO, Cacaulândia* PA, Belém* AM, Fonte Boa (Rothschild 1910); PA, [Belém] (Rothschild 1922)

AM, Fonte Boa (Rothschild 1910), R[io] Juruá (Hampson 1901)

AM, Fonte Boa (Rothschild 1910), Lábrea \{Huitanaã - Rio Purus\} (Bryk 1953), São Paulo de Olivença (Hampson 1901), Tefé (Rothschild 1910); AP, Serra do Navio*; PA, [Belém] (Rothschild 1922), Marabá*; RO, Porto Velho*

AM, Atalaia do Norte \{Santo Antonio do Javari\} (Rothschild 1910); PA, [Belém] (Rothschild 1922); RO, Cacaulândia*

AM, Codajás (Rothschild 1910), Fonte Boa (Rothschild 1909c), [Jutaí] \{Rio Jutahi\} (Rothschild 1909c), Manaus (Rothschild 1909c); MA, Açailândia*; PA, [Belém] (Rothschild 1922)

AM, Fonte Boa (Rothschild 1909c), Rio Purus (Bryk 1953); PA, [Belém] (Rothschild 1922)

AM, Fonte Boa (Rothschild 1909c); PA, Almeirim \{Jari\} (Hawes et al. 2009), [Belém] (Rothschild 1922); RO, Cacaulândia*, Candeias do Jamari*, Porto Velho*

MA, Açailândia*; PA, Marabá*, São Félix do Xingu \{Serra do Pardo National Park\} (Teston and Correa 2015); RO, Ariquemes*, Cacaulândia*

RO, Porto Velho \{Aliança\} (Rothschild 1909b)

AM, Codajás (Rothschild 1910), Fonte Boa (Rothschild 1910)

AM, Benjamin Constant*; PA, Capitão Poço*, Marabá*, São Félix do Xingu \{Serra do Pardo National Park\} (Teston and Correa 2015); RO, Cacaulândia* Porto Velho*

MA, Açailândia*; PA, Prainha (Hampson 1901); RO, Cacaulândia* 
Table 1. Continued.

Species
Carales astur astur (Cramer, [1777])

Carales maculicollis Walker, 1855 Carathis byblis (Schaus, 1892) NEW Castrica phalaenoides (Drury, 1773)

Castrica sordidior Rothschild, 1909 Chrysomallos fulvescens (Rothschild, 1909)

Coiffaitarctia basalis (Rothschild, 1909) Coiffaitarctia coccineata (Rothschild, 1935) Coiffaitarctia ockendeni (Rothschild, 1909) Coiffaitarctia steniptera (Hampson, 1905)

Cratoplastis barrosi (D'Almeida, [1968]) Cratoplastis catherinae (Rothschild, 1916) AMz Cratoplastis diluta Felder \& Rogenhofer, 1874

Cratoplastis rectiradia (Hampson, 1901)

Cresera affinis (Rothschild, 1909)

Cresera hieroglyphica (Schaus, 1905)

Cresera ilioides (Schaus, 1905)

Cresera ilus (Cramer, [1776])

Cresera intensa (Rothschild, 1909) Cresera intermedia (Rothschild, 1922) Cresera optimus (Butler, 1877)

Cresera similis (Rothschild, 1909)

\section{Dialeucias pallidistriata Hampson, 1901}

Diaphanophora albiscripta (Schaus, 1905) Disconeura inexpectata (Rothschild, 1910) Disconeura lutosa frater (Rothschild, 1922) Disconeura peculiaris (Rothschild, 1933) Disconeura soror (Rothschild, 1917) Echeta divisa (Herrich-Schäffer, [1855]) Echeta milesi (Rothschild, 1922) Echeta pandiona (Stoll, [1782]) Echeta rubrireta (Dognin, 1906) NEW Echeta semirosea (Walker, [1865])

Echeta trinotata (Reich, 1933) Elysius amapaensis Rego Barros, 1971
Record

AM, Codajás (Rothschild 1910), Manaus (Travassos 1945a), Santa Isabel do Rio Negro*, São Gabriel da Cachoeira*, São Paulo de Olivença*; AP, Serra do Navio*; MA, Açailândia*; MT, Aripuanã*; PA, Almeirim \{Jari\} (Hawes et al. 2009), Altamira \{Serra do Pardo National Park\} (Teston and Correa 2015), Novo Progresso*, Parauapebas*, São Félix do Xingu \{Serra do Pardo National Park\} (Teston and Correa 2015); RO, Porto Velho (Rothschild 1910) AM, Alto [Rio] Amazonas (Hampson 1901); PA, Novo Progresso*; RO, Cacaulândia* RR, Alto Alegre*

AM, Manaus*; AP, Serra do Navio*; MT, Sinop*; PA, Almeirim \{Jari\} (Hawes et al. 2009), [Belém] (Rothschild 1922), Novo Progresso \{Cachimbo\} (Travassos 1957); RO, Cacaulândia* AM, Fonte Boa (Rothschild 1909a), PA, [Belém] (Rothschild 1922)

AM, Fonte Boa (Rothschild 1909a); AP, Serra do Navio*; MA, Açailândia*; MT, Sinop*; PA, Almeirim \{Jari\} (Hawes et al. 2009), [Belém] (Rothschild 1922), Capitão Poço*; RO, Porto Velho*

AM, Fonte Boa (Rothschild 1909a)

PA, [Belém] (Rothschild 1922)

AM, Fonte Boa (Rothschild 1909a); PA, Almeirim \{Jari\} (Hawes et al. 2009)

AM, Atalaia do Norte \{Santo Antonio do Javari\} (Rothschild 1910), Benjamin Constant*, Fonte Boa (Rothschild 1909a), Manaus*, São Gabriel da Cachoeira*; AP, Serra do Navio*; MT, Sinop*; PA, [Belém] (Rothschild 1922), Benevides*, [Oriximiná] \{Rio Trombetas $\}^{*}$, Tucurí; ${ }^{*}$ RO, Porto Velho*

AP, Serra do Navio (D'Almeida [1968]); MA, Açailândia*; RO, Cacaulândia*, Porto Velho*

PA, Parauapebas*; RO, Cacaulândia*

AM, Fonte Boa (Rothschild 1910), [AM] [Rio] Amazonas (Felder and Rogenhofer 1874); PA, Altamira \{Serra do Pardo National Park\} (Teston and Correa 2015), [Belém] (Hampson 1901), São Félix do Xingu \{Serra do Pardo National Park\} (Teston and Correa 2015)

AM, Benjamin Constant*, Manaus \{Uypiranga\} (Rego Barros 1968), Fonte Boa*, Rio Amazonas (Rothschild 1910), São Gabriel da Cachoeira \{Querari\}*, São Paulo de Olivença (Hampson 1901); AP, Serra do Navio*; MT, Aripuanã*; PA, Almeirim \{Jari\} (Hawes et al. 2009), [Belém] (Rothschild 1922), Novo Progresso \{Cachimbo\} (Rego Barros 1968), Tucuruí; RO, Cacaulândia*, Jaru*

AM, Fonte Boa (Rothschild 1909b), Manaus (Emery 2006), São Gabriel da Cachoeira \{Querari\}*, São Paulo de Olivença (Rego Barros 1958); AP, Serra do Navio (Emery 2006); PA, Almeirim \{Jari\} (Hawes et al. 2009), Capitão Poço (Emery 2006), Marabá (Emery 2006), Santarém (Rego Barros 1958), São Félix do Xingu \{Serra do Pardo National Park\} (Teston and Correa 2015); RO, Vilhena (Emery 2006)

AM, Fonte Boa (Rothschild 1910), [Manaus] \{Estrada Manaus-Itacoatiara - Km26 - Reserva Ducke\} (Emery 2006); AP, Serra do Navio (Emery 2006); PA, Almeirim \{Jari\} (Hawes et al. 2009), Belém (Emery 2006); RO, Vilhena (Emery 2006)

AM, Benjamin Constant (Emery 2006), Fonte Boa (Emery 2006), São Gabriel da Cachoeira \{Querari\}*; AP, Serra do Navio (Emery 2006); MA, Açailândia*; PA, São Félix do Xingu \{Serra do Pardo National Park\} (Teston and Correa 2015); RO, Cacaulândia (Emery 2006), Porto Velho (Emery 2006)

AM, Benjamin Constant (Emery 2006), Fonte Boa (Rothschild 1910), Manaus (Emery 2006), [Novo Airão] \{Jaú National Park - Ig[arapé] Miratuca\} (Emery 2006), [Presidente Figueiredo] \{BR174 - Km173\} (Emery 2006); AP, Serra do Navio (Emery 2006); MT, [Sinop] \{BR163 - Km 500\} (Emery 2006); PA, Almeirim \{Jari\} (Hawes et al. 2009), [Belém] (Rothschild 1922), Marabá*; RO, Porto Velho \{Calam[o]a\} (Rothschild 1910); RR, Alto Alegre (Emery 2006);TO, Ilha do Bananal (Emery 2006)

AM, Atalaia do Norte \{Santo Antonio do Javari\} (Rothschild 1910), Fonte Boa (Rothschild 1909a) PA, [Belém] (Rothschild 1922)

AM, Benjamin Constat (Emery 2006), Fonte Boa (Rothschild 1910), Manaus (Emery 2006), São Paulo de Olivença (Rego Barros 1958), Rio Juruá (Butler 1877); AP, Serra do Navio \{lcomi\} (Emery 2006); MA, Açailândia*; PA, Santarém \{Taperinha\} (Rego Barros 1958), São Félix do Xingu \{Serra do Pardo National Park\} (Teston and Correa 2015); RO, Ariquemes (Emery 2006), Cacaulândia (Emery 2006), Porto Velho (Emery 2006), Vilhena (Emery 2006) AM, Benjamin Constant \{Rio Quichito\} (Rego Barros 1958), Borba \{Lago Acará\} (Rego Barros 1958), Codajás (Rothschild 1909b), Fonte Boa (Rothschild 1909b), Manaus \{Uypiranga\} (Rego Barros 1958), [Novo Airão] \{Jaú National Park - Ig[arapé] Miratuca\} (Emery 2006), [Javari] \{Rio Javari\} (Emery 2006), São Paulo de Olivença (Rego Barros 1958); PA, [Belém] (Rothschild 1922), Marabá (Emery 2006), São Félix do Xingu \{Serra do Pardo National Park\} (Teston and Correa 2015); RO, Ariquemes (Emery 2006), Porto Velho (Emery 2006)

AM, Atalaia do Norte \{Santo Antonio do Javari\} (Rothschild 1910), Fonte Boa (Rothschild 1910), São Paulo de Olivença (Hampson 1901)

AM, Manaus*; PA, Almeirim \{Jari\} (Hawes et al. 2009), [Belém] (Rothschild 1922)

PA, Altamira \{Monte Santo\} (Teston and Delfina 2010)

PA, [Belém] (Rothschild 1922), Ourém*, Viseu*

PA, [Belém] (Rothschild 1933)

AM, [Rio] Amazonas (Rothschild 1917); PA, [Belém] (Watson 1975)

AM, Fonte Boa (Travassos 1943)

PA, [Belém] (Rothschild 1922)

PA, Altamira \{Monte Santo\} (Teston and Delfina 2010), [Belém] (Rothschild 1922)

AM, São Gabriel da Cachoeira \{Querari\}*

AM, Fonte Boa (Rothschild 1910), Rio Amazonas (Rothschild 1910), Tefé (Walker [1865]); MA, Açailândia*; PA, Marabá*; RO, Cacaulândia*

AM, Manaus \{Uypiranga\} (Reich 1933a)

AP, Serra do Navio \{lcomi\} (Rego Barros 1971) 
Table 1. Continued.

\begin{tabular}{l} 
Species \\
\hline Elysius conjunctus Rothschild, $1910^{\mathrm{AMZ}}$ \\
Elysius conspersus Walker, 1855 \\
Elysius disciplaga (Walker, 1856) NEW \\
Elysius hermia (Cramer, [1777]) \\
Elysius intensus Rothschild, 1910 \\
Elysius meridionalis Rothschild, 1917 AMz \\
Elysius pyrosticta Hampson, 1905 AMz \\
Emurena fernandezi Watson, 1975 \\
Emurena lurida (Felder \& Rogenhofer, 187 \\
Eriostepta bacchans Schaus, 1905 NEW \\
Eriostepta roseireta Hampson, 1901 \\
Ernassa cruenta (Rothschild, 1909) \\
Ernassa gabriellae Travassos, 1954 NEW \\
Ernassa sanguinolenta (Cramer, [1779])
\end{tabular}

Eucyrta albicollis Felder \& Rogenhofer, 1874

Eucyrta venusta (Dognin, 1924)

Euplesia sphingidea (Perty, [1833])

Eupseudosoma aberrans Schaus, 1905

Eupseudosoma grandis Rothschild, 1909 AMz Eupseudosoma involuta involuta (Sepp, [1855])

Eupseudosoma larissa (Druce, 1890)

Evius albicoxae (Schaus, 1905)

Evius aurococcinea Walker, 1855

Evius cochenouri Schaus, $1910^{\text {NEW }}$

Evius gabrieli (Bryk, 1953)

Evius hippia (Stoll, [1790])

Glaucostola binotata Schaus, 1905 Glaucostola flavida Schaus, 1905

Glaucostola guttipalpis (Walker, 1856)

Gonotrephes friga (Druce, 1906)

Gorgonidia buckleyi (Druce, 1883)

Gorgonidia garleppi (Druce, 1898) AMZ Gorgonidia harterti (Rothschild, 1909) Gorgonidia pallidipennis (Rothschild, 1910) Gorgonidia vulcania Toulgoët, 1987 Gorgonidia whitfordi (Rothschild, 1909) Graphea pseudomarmorea Travassos, 1956
Record

AM, Manaus*; PA, Belém*, Santarém*

PA, Almeirim \{Jari\} (Hawes et al. 2009), [Belém] (Walker 1855), Novo Progresso \{Cachimbo\} (Rego Barros 1971) MA, Açailândia*

AM, Manaus*; AP, Serra do Navio*; MT, Sinop*; PA, [Belém] (Rothschild 1922), [Rio Tapajós] Itaituba to Óbidos (Rothschild 1910), Novo Progresso \{Cachimbo\}*, Santarém*; RO, Porto Velho \{Aliança\} (Rothschild 1910)

AM, Fonte Boa (Rothschild 1910)

MT, Sinop*; PA, Novo Progresso $\{\text { Cachimbo }\}^{*}$

AP, Serra do Navio*

AM, \{Espirito Santo\} (Watson 1975), Lábrea \{Huitanaã - Rio Purus\} (Watson 1975), São Paulo de Olivença (Watson 1975); MT, Sinop*; PA, Aveiro \{Monte Cristo, Rio Tapajós\} (Watson 1975), [Belém] (Watson 1975), São Félix do Xingu \{Serra do Pardo National Park\} (Teston and Correa 2015); RO, Cacaulândia*

AM, Fonte Boa (Rothschild 1910), Manaus*, [Rio] Amazonas (Felder and Rogenhofer 1874), Rio Purus (Watson 1975); AP, Serra do Navio*; PA, [Belém] (Rothschild 1922); RO, Cacaulândia*

AM, Benjamin Constant*, Fonte Boa* Manaus*, Moura*; AP, Amapari*, Serra do Navio*; PA, Belém*, Benevides*, Marabá*; RO, Porto Velho*

AM, Atalaia do Norte \{Santo Antonio do Javari\} (Rothschild 1910), Fonte Boa (Rothschild 1910), São Paulo de Olivença (Hampson 1901)

AM, Fonte Boa (Rothschild 1909a), Manaus*, São Paulo de Olivença*; AP, Serra do Navio*; MT, Sinop*; PA, [Belém] (Rothschild 1922); RO, Porto Velho*

AP, Serra do Navio*; MT, Sinop*; PA, Novo Progresso $\left\{\right.$ Cachimbo ${ }^{*}$, Viseu*

AM, São Paulo de Olivença*; PA, Belém \{Utinga\} (Travassos 1944a)

AM, Fonte Boa (Rothschild 1910), Manaus*, Tefé (Rothschild 1910); MA, Açailândia*; PA, [Belém] (Rothschild 1922), Óbidos (Travassos 1944a), Parauapebas \{Serra Norte, Carajás\}*, São Félix do Xingu \{Serra do Pardo National Park\} (Teston and Correa 2015), Tucuruí*; RO, Cacaulândia*, Porto Velho*

AM, Fonte Boa (Rothschild 1910), [Rio] Amazonas (Felder and Rogenhofer 1874); PA, Almeirim \{Jari\} (Hawes et al. 2009), Marabá*

PA, Aveiro \{Monte Cristo, Rio Tapajós\} (Dognin 1924), Belém*; RO, Cacaulândia*, Porto Velho*

AM, Benjamin Constant*, Fonte Boa (Rothschild 1910), Itacoatiara*, Manaus*, [Rio] Amazonas (Felder and Rogenhofer 1874), Rio Negro (Perty [1833]), São Paulo de Olivença (Travassos 1944b), Tefé (Butler 1876); AP, Serra do Navio*; MA, Açailândia*; MT, Sinop*; PA, Almeirim \{Jari\} (Hawes et al. 2009), [Belém] (Butler 1876), Novo Progresso \{Cachimbo\} ${ }^{*}$, Óbidos (Rothschild 1910); RO, Ariquemes*, Porto Velho*

AP, Laranjal do Jari (Santos 2006), Serra do Navio*; MA, Açailândia*; PA, Almeirim \{Jari\} (Hawes et al. 2009), Novo Progresso $\{\text { Cachimbo }\}^{*}$, Santarém*

AM, Fonte Boa*; AP, Serra do Navio*; PA, Belém*

AM, Fonte Boa (Rothschild 1910), Manaus*; AP, Laranjal do Jari (Santos 2006); MA, Açailândia*; PA, Almeirim \{Jari\} (Hawes et al. 2009), Altamira \{Monte Santo\} (Teston and Delfina 2010), [Belém] (Rothschild 1922), Santarém (Travassos 1945b), São Félix do Xingu \{Serra do Pardo National Park\} (Teston and Correa 2015); RO, Ariquemes*, Porto Velho*

AM, Benjamin Constant* Fonte Boa (Rothschild 1910), Rio Madeira (Rothschild 1922), Tefé*; MT, Sinop*; PA, Almeirim \{Jari\} (Hawes et al. 2009), [Belém] (Rothschild 1922), Breves*, Santarém (Druce 1890), São Félix do Xingu \{Serra do Pardo National Park\} (Teston and Correa 2015); RO, Cacaulândia*, Porto Velho*

AM, Benjamin Constant*, Fonte Boa (Rothschild 1909b), Manaus*, São Gabriel da Cachoeira \{Querari\}*, Tefé (Rothschild 1909b); AP, Serra do Navio*; PA, Almeirim \{Jari\} (Hawes et al. 2009), Altamira \{Serra do Pardo National Park\} (Teston and Correa 2015), Belém*, São Félix do Xingu \{Serra do Pardo National Park\} (Teston and Correa 2015), Viseu*

AM, Fonte Boa (Rothschild 1910); PA, [Belém] (Walker 1855)

RO, Porto Velho*

AM, São Gabriel [da Cachoeira] (Bryk 1953)

AM, Benjamin Constant*, Fonte Boa (Rothschild 1910), Maués*, Manaus \{Uypiranga\}*, [Rio] Amazonas (Rothschild 1910), Rio Solimões (Bryk 1953), Tefé (Bryk 1953); AP, Serra do Navio*; MA, Açailândia*; PA, Almeirim \{Jari\} (Hawes et al. 2009), [Belém] (Druce 1890), Marabá*; RO, Cacaulândia*

AM, Fonte Boa (Rothschild 1910); RO, Cacaulândia*

AM, Rio Purus (Bryk 1953); AP, Serra do Navio*; MA, Açailândia*; PA, [Belém] (Rothschild 1922); RO, Ariquemes*, Cacaulândia*

AM, Fonte Boa (Rothschild 1910), Manaus*, Moura*; MA, Açailândia*; RO, Cacaulândia*

AM, Benjamin Constant*, Fonte Boa (Rothschild 1910); PA, [Belém] (Rothschild 1922); RO, Candeias do Jamari*

AM, Benjamin Constant*, Borba*, Manaus*, Presidente Figueiredo*; AP, Serra do Navio*; MA, Açailândia*; PA, Almeirim \{Jari\} (Hawes et al. 2009), São Félix do Xingu \{Serra do Pardo National Park\} (Teston and Correa 2015); RO, Cacaulândia*, Candeias do Jamari*, Porto Velho*

RO, Cacaulândia*, Jaru*, Porto Velho*

AM, Fonte Boa (Rothschild 1909a)

AM, Fonte Boa (Rothschild 1910)

PA, Belterra \{Tapajós National Forest\} (Teston and Freitas 2015)

PA, Belterra \{Tapajós National Forest\} (Teston and Freitas 2015)

AM, Benjamin Constant (Travassos 1956), [Rio] Amazonas (Travassos 1956); MT, Sinop*; PA, Novo Progresso \{Cachimbo\} (Travassos 1956) 
Table 1. Continued.

\begin{tabular}{l} 
Species \\
\hline Haemanota abdominalis Rothschild, 1909 \\
Haemanota affinis (Rothschild, 1909) \\
Haemanota griseotincta (Rothschild, 1909) \\
Haemanota holophaea (Hampson, 1905) \\
Haemanota improvisa (Dognin, 1923) \\
Haemanota nigricollum (Dognin, 1892) ${ }^{\mathrm{NEW}}$ \\
Haemanota rubriceps Hampson, 1901 \\
Haemanota sanguidorsia (Schaus, 1905) NEW \\
Haemaphlebiella formona (Schaus, 1905) \\
Haemaphlebiella strigata Jones, 1914 ${ }^{\mathrm{NEW}}$ \\
Haemaphlebiella venata (Rothschild, 1909)
\end{tabular}

Halysidota baritioides (Rothschild, 1909) Halysidota interlineata (Walker, 1855)

Halysidota orientalis (Rothschild, 1909) Halysidota pectenella Watson, 1980 Himerarctia docis (Hübner, [1831])

Himerarctia griseipennis (Rothschild, 1909)

Himerarctia laeta Watson, 1975

Himerarctia viridisignata Watson, 1975

Hyalarctia sericea Schaus, 1901 NEW

Hyperandra appendiculata (Herrich-Schäffer, [1856])

Hyperandra diminuta Dognin, 1923

Hyperandra novata (Dognin, 1924)

Hyperthaema hoffmannsi Rothschild, 1909 Hypidalia sanguirena sanguirena Schaus, 1905 NEW

Hyponerita lavinia (Druce, 1890) NEW Hyponerita persimilis Rothschild, 1909 Hyponerita similis Rothschild, 1909

Idalus admirabilis (Cramer, [1777])

Idalus aleteria (Schaus, 1905)

Idalus carinosa (Schaus, 1905)

Idalus citrina Druce, 1890

Idalus critheis Druce, 1884

Idalus daga (Dognin, 1891)

Idalus dares Druce, 1894

Idalus fasciipuncta (Rothschild, 1909)

Idalus felderi (Rothschild, 1909) NEW

Idalus herois Schaus, 1889

Idalus intermedia (Rothschild, 1909)

Idalus lineosus Walker, 1869 NEW

Idalus metacrinis (Rothschild, 1909)

\section{Record}

AM, Fonte Boa(Rothschild 1909c)

AM, Fonte Boa (Rothschild 1909b); MA, Açailândia*

RO, Cacaulândia*

AM, Fonte Boa (Rothschild 1910); PA, Capitão Poço*; RO, Porto Velho*

AM, Benjamin Constant*, São Paulo de Olivença (Dognin 1923c); PA, Almeirim \{Jari\} (Hawes et al. 2009)

RO, Cacaulândia*

PA, [Belém] (Rothschild 1922)

MA, Açailândia*; RO, Cacaulândia*

AM, Fonte Boa (Rothschild 1910), Manaus*; AP, Serra do Navio*

PA, Itaituba \{Amazônia National Park\}

AM, Fonte Boa (Rothschild 1909c), São Gabriel da Cachoeira \{Querari\}*; AP, Serra do Navio*; RO, Ariquemes* Porto Velho*

AM, Fonte Boa(Rothschild 1909c)

AP, Serra do Navio*; MT, Sinop*; PA, Almeirim \{Jari\} (Hawes et al. 2009), Belém*, Novo Progresso \{Cachimbo\} (Travassos 1963), Parauapebas \{Serra Norte, Carajás\}*, Santarém*, Tucuruí*

PA, [Belém] (Watson 1980), Óbidos (Watson 1980)

PA, [Belém] (Watson 1980)

AM, Atalaia do Norte \{Santo Antonio do Javari\} (Rothschild 1909a), Benjamin Constant* Borba*, Fonte Boa (Rothschild 1909a), São Paulo de Olivença (Watson 1975), Vale do [Rio] Amazonas (Walker 1856); AP, Serra do Navio*; PA, [Rio Tapajós] Itaituba to Óbidos (Rothschild 1909a), Óbidos (Rothschild 1909a), Parauapebas \{Serra Norte, Carajás\} ${ }^{*}$; RO, Candeias do Jamari* ${ }^{*}$ Porto Velho*

AM, Fonte Boa (Rothschild 1909a), Manaus*, São Paulo de Olivença*; MA, Açailândia*; PA, Parauapebas \{Serra Norte, Carajás\} ${ }^{*}$, Tucuruí*; RO, Porto Velho*

AM (Seitz 1921), Atalaia do Norte \{Santo Antonio do Javari\} (Watson 1975), Fonte Boa (Watson 1975), Parintins (Watson 1975), São Paulo de Olivença (Watson 1975), PA, Óbidos (Watson 1975); RO, Porto Velho* AM, Fonte Boa (Watson 1975)

AM, Manaus*

AM, Benjamin Constant*, Fonte Boa (Rothschild 1910), Manaus*; MT, Sinop*; PA, Marabá*, Novo Progresso $\{$ Cachimbo\}*; RO, Cacaulândia*

AM, São Paulo de Olivença (Dognin 1923a)

AP, Serra do Navio (D’Almeida 1968); MA, Açailândia*; PA, Altamira \{Monte Santo\} (Teston and Delfina 2010), São Félix do Xingu \{Serra do Pardo National Park\} (Teston and Correa 2015), Vitória do Xingu \{Ponte Nova\} (Dognin 1924)

AM, Tefé (Rothschild 1909c); RO, Porto Velho \{Aliança\} (Rothschild 1909c)

AM, Manaus*; RO, Cacaulândia*

AM, Fonte Boa*, Manaus*; AP, Serra do Navio*; RO, Cacaulândia*, Porto Velho*

AM, Fonte Boa (Rothschild 1909b), Rio Negro \{Alto\} (Seitz 1921)

AM, Benjamin Constant*, Fonte Boa (Rothschild 1909b), Manaus*; AP, Serra do Navio*; PA, [Belém] (Rothschild 1922), Marabá*, São Félix do Xingu \{Serra do Pardo National Park\} (Teston and Correa 2015)

AM, Benjamin Constant* Fonte Boa (Rothschild 1910), [Rio] Amazonas (Rothschild 1910); AP, Laranjal do Jari (Santos 2006); MA, Açailândia*; PA, Almeirim \{Jari\} (Hawes et al. 2009), Altamira \{Monte Santo\} (Teston and Delfina 2010), [Belém] (Rothschild 1922), Marabá*; RO, Cacaulandia*, Porto Velho*

AM, Borba*, Fonte Boa (Rothschild 1910), Manaus*; MA, Açailândia*, Itinga do Maranhão*; PA, Almeirim \{Jari\} (Hawes et al. 2009), [Belém] (Rothschild 1922), Parauapebas \{Serra Norte, Carajás\} ${ }^{*}$, São Félix do Xingu \{Serra do Pardo National Park\} (Teston and Correa 2015); RO, Cacaulândia*, Candeias do Jamari*, Porto Velho*

AM, Benjamin Constant (Travassos 1950), Fonte Boa*, Manaus*; AP, Serra do Navio*; PA, São Félix do Xingu \{Serra do Pardo National Park\} (Teston and Correa 2015); RO, Cacaulândia*

PA, Altamira \{Monte Santo\} (Teston and Delfina 2010)

AM, Benjamin Constant*, Fonte Boa (Rothschild 1910), Manaus (Hampson 1901); MA, Açailândia*; PA, Altamira \{Monte Santo\} (Teston and Delfina 2010) and \{Serra do Pardo National Park\} (Teston and Correa 2015), [Belém] (Rothschild 1922), Parauapebas \{Serra Norte, Carajás\}*, São Félix do Xingu \{Serra do Pardo National Park\} (Teston and Correa 2015); RO, Cacaulândia*, Candeias do Jamari*

AM, Atalaia do Norte \{Santo Antonio do Javari\} (Rothschild 1910), São Paulo de Olivença (Hampson 1901); PA, [Belém] (Rothschild 1922), São Félix do Xingu \{Serra do Pardo National Park\} (Teston and Correa 2015)

AM, São Paulo de Olivença (Travassos 1952); AP, Serra do Navio*; PA, Belém*; RO, Cacaulândia*

AM, Fonte Boa (Rothschild 1909a); PA, São Félix do Xingu \{Serra do Pardo National Park\} (Teston and Correa 2015); RO, Cacaulândia*

AM, Fonte Boa*, São Paulo de Olivença*; PA, Parauapebas \{Serra Norte, Carajás\}*

AM, Benjamin Constant* ${ }^{*}$ Fonte Boa (Rothschild 1910), Manaus (Travassos 1950), São Paulo de Olivença (Travassos 1950); PA, Óbidos*; RO, Cacaulândia*

[AM] [Rio] Amazonas (Rothschild 1910); PA, [Belém] (Rothschild 1922), São Félix do Xingu \{Serra do Pardo National Park\} (Teston and Correa 2015); RO, Porto Velho \{Aliança\} (Rothschild 1910)

AM, Fonte Boa*, Tabatinga*; AP, Amapari*, Serra do Navio*; PA, Novo Progresso \{Cachimbo\} ${ }^{*}$, Viseu*; RO, Cacaulândia*

AP, Serra do Navio*; PA, Almeirim \{Jari\} (Hawes et al. 2009) 
Table 1. Continued.

\begin{tabular}{l} 
Species \\
\hline Idalus mossi (Rothschild, 1922) \\
Idalus nigropunctata (Rothschild, 1909) \\
Idalus ochracea (Rothschild, 1909) \\
Idalus tybris (Cramer, [1776]) \\
Idalus vitrea vitrea (Cramer, [1780]) \\
Idalus vitreoides (Rothschild, 1922) \\
Ischnognatha semiopalina Felder \& Rogenhofer, \\
1874
\end{tabular}

Isia alcumena alcumena (Berg, 1882) NEW Lepidokirbyia vittipes vittipes (Walker, 1855)

Lepidokirbyia venigera Toulgoët, [1983] Lepidolutzia baucis (Dalman, 1823) Leucanopsis aurantiaca (Rothschild, 1909) Leucanopsis batesi (Rothschild, 1909) Leucanopsis cedon (Druce, 1897) Leucanopsis contempta (Rothschild, 1909) Leucanopsis curta (Rothschild, 1910) Leucanopsis hadenoides (Rothschild, 1909) Leucanopsis jonesi (Rothschild, 1909) NEW Leucanopsis liparoides (Rothschild, 1909) Leucanopsis maccessoya (Schaus, 1933) Leucanopsis nebulosa (Rothschild, 1909) Leucanopsis ochracea (Möschler, 1883) Leucanopsis polyodonta (Hampson, 1901) Leucanopsis pulverulenta (Dognin, 1923) Leucanopsis rhomboidea (Sepp, [1852]) Leucanopsis rufoochracea (Rothschild, 1922) Leucanopsis similis (Rothschild, 1909) Leucanopsis squalida (Herrich-Schäffer, [1855])

Leucanopsis stipulata (Rothschild, 1909) Leucanopsis stipulatoides (Rothschild, 1910) Leucanopsis strigulosa (Walker, 1855) Leucanopsis subnebulosa (Strand, 1919) Leucanopsis terranea (Rothschild, 1909) Leucanopsis umbrina (Rothschild, 1910) Lophocampa aenone (Butler, 1878)

Lophocampa amaxiaeformis (Rothschild, 1910) Lophocampa annulosa (Walker, 1855)

Lophocampa catenulata (Hübner, [1812]) Lophocampa citrina (Sepp, [1852])

Lophocampa endolobata (Hampson, 1901)

Lophocampa endrolepia (Dognin, 1908) Lophocampa maroniensis buchwaldi (Rothschild 1910)

Lophocampa modesta (Kirby, 1892)

Lophocampa pectina (Schaus, 1896)
Record

PA, [Belém] (Rothschild 1922)

AM, Fonte Boa (Rothschild 1909a), Tefé (Vincent and Laguerre 2014); PA, Almeirim \{Jari\} (Hawes et al. 2009)

AM, Benjamin Constant (Travassos 1950), Manaus \{lpiranga\} (Travassos 1950), São Paulo de Olivença (Travassos 1950); PA, Belém \{Utinga\} (Travassos 1950)

AM, Benjamin Constant*, Fonte Boa (Rothschild 1910), Moura*, Rio Amazonas (Rothschild 1910), São Paulo de Olivença*, Tefé*; AP, Amapari*; MA, Açailândia*; PA, Novo Progresso \{Cachimbo\}*, Óbidos*; RO, Porto Velho*

AM, Fonte Boa (Rothschild 1910), Manaus*, Moura*; AP, Amapari*, Serra do Navio*; MA, Açailândia*; MT, Sinop*; PA, [Belém] (Rothschild 1922), Marabá*, Novo Progresso \{Cachimbo\}*, São Félix do Xingu \{Serra do Pardo National Park\} (Teston and Correa 2015), Viseu*; RO, Cacaulândia*, Candeias do Jamari*, Porto Velho*

PA, [Belém] (Rothschild 1922)

AM, Benjamin Constant*, Fonte Boa (Rothschild 1910), São Paulo de Olivença*; AP, Serra do Navio*; MA, Açailândia*; PA, Almeirim \{Jari\} (Hawes et al. 2009), [Belém] (Rothschild 1922), São Félix do Xingu \{Serra do Pardo National Park\} (Teston and Correa 2015); RO, Cacaulândia*, Porto Velho*

RO, Cacaulândia*

AM, Fonte Boa (Rothschild 1910), Humaitá*, Rio Amazonas (Rothschild 1910); AP, Serra do Navio*; MA, Açailândia*; PA, Almeirim \{Jari\} (Hawes et al. 2009), Altamira \{Monte Santo\} (Teston and Delfina 2010), [Belém] (Walker 1855), Marabá*, Parauapebas \{Serra Norte, Carajás\} ${ }^{*}$, [Santarém] (Walker 1855), Santarém (Hampson 1901); RO, Cacaulândia*, Porto Velho*

São Félix do Xingu \{Serra do Pardo National Park\} (Teston and Correa 2015)

PA, [Belém] (Hampson 1901)

MA, Açailândia*; PA, Marabá*; RO, Porto Velho \{Aliança\} (Rothschild 1909b)

AM, Tefé (Rothschild 1909b); PA, Marabá*; RO, Jaru*

RO, Porto Velho \{Aliança\} (Rothschild 1910)

AM, Fonte Boa (Rothschild 1909b)

AM, Fonte Boa (Rothschild 1910)

AM, Codajás (Rothschild 1909b), Fonte Boa (Rothschild 1909b); RO, Porto Velho \{Aliança\} (Rothschild 1909b)

MA, Açailândia*

AM, Fonte Boa (Rothschild 1909b)

AM, Amaturá (Schaus 1933)

AM, Fonte Boa (Rothschild 1909b)

AM, Fonte Boa (Rothschild 1910); PA, [Belém] (Rothschild 1922)

AM, Parintins (Hampson 1901); PA, [Belém] (Rothschild 1922)

AM, São Paulo de Olivença (Dognin 1923c); PA, Rio Tapajós (Dognin 1923c)

PA, [Belém] (Hampson 1901)

PA, [Belém] (Rothschild 1922)

AM, Fonte Boa (Rothschild 1909c); PA, Marabá*; RO, Cacaulândia*

AM, Atalaia do Norte \{Santo Antonio do Javari\} (Rothschild 1910), Fonte Boa (Rothschild 1910); MA, Açailândia*;

PA, Óbidos (Rothschild 1910)

RO, Porto Velho \{Aliança\} (Rothschild 1910)

AM, Codajás (Rothschild 1910), Fonte Boa (Rothschild 1910); PA, [Belém] (Rothschild 1922)

PA, [Belém] (Walker 1855)

AM, Fonte Boa (Rothschild 1910); RO, Porto Velho \{Aliança and Calama\} (Rothschild 1910)

AM, Fonte Boa (Rothschild 1909c)

AM, Fonte Boa (Rothschild 1910)

AM, Atalaia do Norte \{Santo Antonio do Javari\} (Rothschild 1910), Codajás (Rothschild 1910), Fonte Boa (Rothschild 1910), Lábrea \{[S]epatiny e Mabid[e]r[i], Rio Purús\} (Butler 1878), Manaus to Yutahi [Jutaí] (Rothschild 1910), Rio Juruá \{Pupunha, Prainha\} (Butler 1878), Rio Purus \{Pedroso, Urucuri\} (Butler 1878), Rio Solimões \{llha Cuxinara\} (Butler 1878); PA, [Belém] (Rothschild 1922), [Rio Tapajós] Itaituba to Óbidos (Rothschild 1910)

PA, Santarém (Rothschild 1922)

AM, Fonte Boa (Rothschild 1910); MA, Açailândia*; PA, [Belém] (Walker 1855); RO, Porto Velho \{Aliança\} (Rothschild 1910)

[AM], Alto Amazonas (Bryk 1953)

AM, Rio Madeira (Rothschild 1922), Rio Purus (Hampson 1901); AP, Serra do Navio*; MA, Açailândia*; PA, Altamira \{Serra do Pardo National Park\} (Teston and Correa 2015), [Belém] (Hampson 1901), São Félix do Xingu \{Serra do Pardo National Park\} (Teston and Correa 2015); RO, Cacaulândia*

AM, Atalaia do Norte \{Santo Antonio do Javari\} (Rothschild 1910), Fonte Boa (Rothschild 1910), [Rio] Solimões (Bryk 1953), [São Paulo de] Olivença (Hampson 1901), Tefé (Rothschild 1910)

[PA] Baixo Amazonas (Rothschild 1922)

AM, Atalaia do Norte \{Santo Antonio do Javari\} (Rothschild 1910), Fonte Boa (Rothschild 1910); PA, [Belém] (Rothschild 1922)

AM, Benjamin Constant*, Fonte Boa (Rothschild 1910), Tefé (Rothschild 1910); MA, Açailândia*; PA, Altamira \{Monte Santo\} (Teston and Delfina 2010), [Belém] (Rothschild 1922), Marabá*, São Félix do Xingu \{Serra do Pardo National Park\} (Teston and Correa 2015); RO, Cacaulândia*

AM, Manaus*, Moura*; MT, Aripuanã*; PA, Almeirim \{Jari\} (Hawes et al. 2009) 
Table 1. Continued.

\begin{tabular}{|c|c|}
\hline Species & Record \\
\hline Lophocampa russus (Rothschild, 1909) & $\begin{array}{l}\text { AM, Atalaia do Norte \{Santo Antonio do Javari\} (Rothschild 1910), Codajás (Rothschild 1910), Fonte Boa } \\
\text { (Rothschild 1910); PA, [Belém] (Rothschild 1922) }\end{array}$ \\
\hline Lophocampa secunda Vincent, 2009 & AM, Fonte Boa (Rothschild 1909c) \\
\hline Lophocampa seruba (Herrich-Schäffer, [1855]) & $\begin{array}{l}\text { AM, Fone Boa (Rothschild 1910), [Rio] Amazonas (Rothschild 1910), Rio Purus \{Mamiva\} (Butler 1878); RO, Porto } \\
\text { Velho \{Aliança\} (Rothschild 1910) }\end{array}$ \\
\hline Lophocampa sesia (Sepp, [1852]) & AM, Codajás (Rothschild 1910), Fonte Boa (Rothschild 1910) \\
\hline Lophocampa sobrinoides (Rothschild, 1910) & AM, Fonte Boa (Rothschild 1910) \\
\hline Lophocampa subannula (Schaus, 1911) NEW & PA, Marabá* \\
\hline Lophocampa subvitreata (Rothschild, 1922) & PA, [Belém] (Rothschild 1922) \\
\hline Lophocampa teffeana (Schaus, 1933) & AM, Tefé (Schaus 1933) \\
\hline Lophocampa testacea (Möschler, 1878) & AM, Rio Juruá (Hampson 1901), Rio Purus (Hampson 1901); [PA], Baixo Amazonas (Hampson 1901) \\
\hline Machadoia diminuta (Walker, 1855) AMz & PA, Belém* \\
\hline Machaeraptenus crocopera (Schaus, 1905) & $\begin{array}{l}\text { AP, Serra do Navio*; PA, Almeirim \{Jari\} (Hawes et al. 2009), [Belém] (Rothschild 1922); RO, Cacaulândia*, Candeias } \\
\text { do Jamari* }\end{array}$ \\
\hline Mazaeras conferta Walker, 1855 AMz & MA, Açailândia* \\
\hline Mazaeras francki Schaus, 1896 & AM, [Rio] Amazonas (Reich 1937b); RO, Cacaulândia* \\
\hline Mazaeras magnifica (Rothschild, 1909) NEW & AP, Serra do Navio* \\
\hline Melanarctia lativitta (Rothschild, 1909) & AM, Fonte Boa (Rothschild 1909a), Manicoré \{Rio Madeira\} (Watson 1975) \\
\hline Melese asana Druce, 1884 & AM, Fonte Boa (Rothschild 1910), Rio Amazonas (Rothschild 1910), Tefé (Hampson 1901); MA, Açailândia* \\
\hline Melese babosa (Dognin, 1894) NEW & MA, Açailândia*; RO, Cacaulândia* \\
\hline Melese chozeba (Druce, 1884) & AM, Fonte Boa (Rothschild 1910) \\
\hline Melese dorothea (Stoll, [1782]) & AP, Serra do Navio*; MT, Sinop*; PA, [Belém] (Rothschild 1922) \\
\hline Melese drucei Rothschild, 1909 & $\begin{array}{l}\text { AM, Fonte Boa (Rothschild 1909b); PA, Almeirim \{Jari\} (Hawes et al. 2009), Altamira \{Monte Santo\} (Teston and } \\
\text { Delfina 2010) and \{Serra do Pardo National Park\} (Teston and Correa 2015), São Félix do Xingu \{Serra do Pardo } \\
\text { National Park\} (Teston and Correa 2015); RO, Cacaulândia*, Porto Velho* }\end{array}$ \\
\hline Melese hampsoni Rothschild, 1909 & AM, Fonte Boa (Rothschild 1909b); RO, Cacaulândia*, Porto Velho* \\
\hline Melese hebetis Rothschild, 1909 Amz & MA, Açailândia*; RO, Cacaulândia* \\
\hline Melese incertus (Walker, 1855) & $\begin{array}{l}\text { AM, Fonte Boa (Rothschild 1909a), São Gabriel da Cachoeira \{Querari\}*; AP, Serra do Navio*; MA, Açailândia*; PA, } \\
\text { Altamira \{Monte Santo\} (Teston and Delfina 2010), [Belém] (Walker 1855), Castanhal*, São Félix do Xingu \{Serra } \\
\text { do Pardo National Park\} (Teston and Correa 2015); RO, Ariquemes*, Cacaulândia*, Candeias do Jamari* }\end{array}$ \\
\hline Melese inconspicua Rothschild, 1909 & AM, Fonte Boa (Rothschild 1909a) \\
\hline Melese ocellata Hampson, 1901 & PA, São Félix do Xingu \{Serra do Pardo National Park\} (Teston and Correa 2015); RO, Cacaulândia*, Porto Velho* \\
\hline Melese postica Walker, 1854 & $\begin{array}{l}\text { AM, Atalaia do Norte \{Santo Antonio do Javari\} (Rothschild 1910), Fonte Boa (Rothschild 1910), Rio Amazonas } \\
\text { (Rothschild 1910), Tefé (Hampson 1901); PA, [Belém] (Walker 1854b), Santarém \{Vila Nova\} (Hampson 1901) }\end{array}$ \\
\hline Melese pumila Dognin, 1908 & AM, Codajás (Rothschild 1910), Fonte Boa (Rothschild 1910) \\
\hline Melese pusilla Rothschild, 1909 & AM, Fonte Boa (Rothschild 1909b), Tefé (Hampson 1920) \\
\hline Metaxanthia threnodes Druce, 1905 & $\begin{array}{l}\text { AM, Santa Isabel do Rio Negro*; MT, Sinop*; PA, Santarém*, São Félix do Xingu \{Serra do Pardo National Park\} } \\
\text { (Teston and Correa 2015) }\end{array}$ \\
\hline Metaxanthia vespiformis Druce, 1899 & AM, Manaus*; PA, [Belém] (Rothschild 1922), Santarém \{Vila Nova\} (Druce 1899) \\
\hline Munona iridescens Schaus, 1894 & AP, Serra do Navio*; PA, Altamira \{Serra do Pardo National Park\} (Teston and Correa 2015); RO, Cacaulândia* \\
\hline Neidalia bipuncta Rothschild, 1922 & PA, [Belém] (Rothschild 1922) \\
\hline Neidalia bifasciata (Cramer, [1779]) & AM, Fonte Boa (Rothschild 1910); MA, Açailândia*; PA, [Belém] (Rothschild 1922); RO, Porto Velho* \\
\hline Neonerita dorsipuncta Hampson, 1901 & $\begin{array}{l}\text { [AM], Amazonas (Travassos 1945b); MA, Açailândia*; PA, Almeirim \{Jari\} (Hawes et al. 2009); RO, Cacaulândia*, } \\
\text { Porto Velho \{Calama\} (Rothschild 1910) }\end{array}$ \\
\hline Neonerita fenestrata fenestrata (Rothschild, 1910) & [AM] Rio Amazonas (Rothschild 1910) \\
\hline Neozatrephes schausi (Rothschild, 1909) & AM, Fonte Boa (Rothschild 1910); RO, Porto Velho \{Aliança\} (Rothschild 1910) \\
\hline Neozatrephes telesilla Druce, $1893^{\mathrm{NEW}}$ & AM, Fonte Boa*; PA, Marabá* \\
\hline Nezula grisea Schaus, 1896 & AM, Fonte Boa (Rothschild 1910); RO, Cacaulândia* \\
\hline Nyearctia leucoptera (Hampson, 1920) & AP, Amapari*, Serra do Navio*; PA, [Belém] (Rothschild 1922); RO, Cacaulândia* \\
\hline Ochrodota affinis Rothschild, 1909 & AM, Codajás (Rothschild 1909c), Fonte Boa (Rothschild 1909c), Tefé (Rothschild 1910) \\
\hline Ochrodota brunnescens Rothschild, 1909 & AM, Codajás (Rothschild 1910), Fonte Boa (Rothschild 1909c); PA, [Belém] (Rothschild 1922) \\
\hline Ochrodota funebris Rothschild, $1909^{\mathrm{NEW}}$ & AM, Benjamin Constant*; RO, Cacaulândia* \\
\hline Ochrodota pronapides (Druce, 1894) & AM, Codajás (Rothschild 1910), Fonte Boa (Rothschild 1910); PA, [Belém] (Rothschild 1922); RO, Porto Velho* \\
\hline Ochrodota similis Rothschild, 1909 & $\begin{array}{l}\text { AM, Atalaia do Norte \{Santo Antonio do Javari\} (Rothschild 1910), Codajás (Rothschild 1909c), Fonte Boa } \\
\text { (Rothschild 1909c) }\end{array}$ \\
\hline Ochrodota tessellata Rothschild, 1909 & AM, Fonte Boa (Rothschild 1909c); PA, [Belém] (Rothschild 1922), Viseu* \\
\hline Opharus bimaculata (Dewitz, 1877) NEW & RO, Cacaulândia* \\
\hline Opharus flavimaculata Hampson, 1901 & PA, [Rio] Tapajós (Hampson 1901) \\
\hline
\end{tabular}


Table 1. Continued.

\begin{tabular}{l}
\hline Species \\
\hline Ordishia klagesi (Rothschild, 1909) \\
Ordishia rutilus (Stoll, [1782]) \\
Ormetica albimaculifera (Hampson, 1901) \\
Ormetica ataenia (Schaus, 1910) \\
Ormetica bonora (Schaus, 1905) \\
Ormetica contraria contraria (Walker, 1854)
\end{tabular}

Ormetica neira (Schaus, 1905) AMz

Ormetica orbona (Schaus, 1889)

Ormetica packardi (Buter, 1876)

Ormetica rothschildi Watson, $1975^{\mathrm{NEW}}$

Ormetica stenotis (Dognin, 1908)

Ormetica sypilus (Cramer, [1777])

Ormetica xanthia (Hampson, 1901)

Ormetica zenzeroides (Butler, 1877)

Pachydota albiceps (Walker, 1856)

Pachydota punctata Rothschild, 1909

Paranerita gaedei Reich, 1933

Parathyris cedonulli (Stoll, [1781])

Pareuchaetes aurata (Butler, 1875)

Pareuchaetes bipunctata (Walker, 1855)

Pareuchaetes insulata (Walker, 1855)

Pelochyta arontes (Stoll, [1782])

Phaeomolis brunnescens brunnescens (Rothschild, 1909)

Phaeomolis obnubila Dognin, 1923

Phaeomolis obscurata (Butler, 1877)

Phaeomolis polystria (Schaus, 1905)

Premolis schausi (Rothschild, 1909)

Premolis semirufa (Walker, 1856)

Pryteria alboatra alboatra (Rothschild, 1909)

Pryteria semicostalis (Rothschild, 1909)

Pseudepimolis apiciplaga (Rothschild, 1909)

Pseudepimolis flavonotata (Rothschild, 1909)

Pseudepimolis incarnata (Hampson, 1901)

Pseudepimolis incisa (Rothschild, 1909)

Pseudepimolis marpessa (Druce, 1906)

Pseudepimolis ridenda (Dognin 1911) NEW

Pseudischnocampa nervosa (Felder \&

Rogenhofer, 1874) NEW
Record

AM, Codajás (Rothschild 1910), Fonte Boa (Rothschild 1909a); PA, [Belém] (Watson 1975)

AM, Tefé (Rothschild 1910); AP, Serra do Navio*; MA, Açailândia*; PA, [Belém] (Rothschild 1922); RO, Cacaulândia*

AM, Fonte Boa (Rothschild 1910), Manicoré (Hampson 1901), Tefé (Rothschild 1910)

AM, Rio Negro (Seitz 1921); PA, [Belém] (Seitz 1921)

MA, Açailândia*; PA, [Belém] (Rothschild 1922)

AM, Benjamin Constant*, Borba*, Fonte Boa (Rothschild 1910), Humaitá (Rothschild 1910), Tefé (Walker 1854a); AP, Serra do Navio*; MA, Açailândia*; MT, Aripuanã*; PA, [Belém] (Rothschild 1922), Benevides*, Marabá*,

Parauapebas \{Serra Norte, Carajás\}*, São Félix do Xingu \{Serra do Pardo National Park\} (Teston and Correa 2015);

RO, Cacaulândia*, Jaru*, Porto Velho*; RR, Alto Alegre*

AM, Benjamin Constant*; AP, Serra do Navio*; PA, Óbidos*

PA, [Belém] (Rothschild 1922)

AM, Fonte Boa (Rothschild 1910), Humaitá (Rothschild 1910), Tefé (Butler, 1876); PA, Altamira \{Serra do Pardo

National Park\} (Teston and Correa 2015), [Belém] (Butler 1876), [Rio Tapajós] Itaituba to Óbidos (Rothschild 1910), São Félix do Xingu \{Serra do Pardo National Park\} (Teston and Correa 2015)

AP, Serra do Navio*; PA, Oriximiná \{Rio Cuminá\}*; RO, Cacaulândia*, Jaru*, Porto Velho*

AM, Benjamin Constant*, Fonte Boa (Rothschild 1910)

AM, Fonte Boa (Rothschild 1910), Manaus*, [Rio] Amazonas (Felder and Rogenhofer 1874); MA, Açailândia*; PA, Altamira \{Monte Santo\} (Teston and Delfina 2010), [Belém] (Walker 1856), Parauapebas \{Serra Norte, Carajás\}*,

São Félix do Xingu \{Serra do Pardo National Park\} (Teston and Correa 2015); RO, Cacaulândia*, Jaru*, Porto Velho \{Aliança\} (Rothschild 1910)

PA, [Belém] (Rothschild 1922)

AM, Benjamin Constant*, Fonte Boa (Rothschild 1910), R[io] Juruá (Watson 1975), Rio Purus \{Porto Salvo\} (Butler 1877); AP, Serra do Navio*; RO, Cacaulândia*, Candeias do Jamari*, Porto Velho*

AP, Serra do Navio*; PA, Santarém*, São Félix do Xingu \{Serra do Pardo National Park\} (Teston and Correa 2015)

PA, Belém*, Parauapebas \{Serra Norte, Carajás\}*, Santarém*, Tucuruí*

AM, Manaus \{Uypiranga\} (Reich 1933)

AM, Benjamin Constant (Travassos 1949), Fonte Boa (Rothschild 1910), São Paulo de Olivença (Travassos 1949); AP, Serra do Navio*; PA, Almeirim \{Jari\} (Hawes et al. 2009), [Belém] (Rothschild 1922), São Félix do Xingu \{Serra do Pardo National Park\} (Teston and Correa 2015); RO, Porto Velho*

AM, Tefé (Rego Barros 1956); MA, Açailândia*; PA, Altamira \{Monte Santo\} (Teston and Delfina 2010), [Belém] (Rothschild 1922), Capitão Poço*, Santarém (Hampson 1901); RO, Cacaulândia*

PA, [Belém] (Walker 1855)

AM Manaus (Hampson 1901); AP, Laranjal do Jari (Santos 2006); MA, Açailândia*; PA, Almeirim \{Jari\} (Hawes et al. 2009), Santarém (Walker 1855)

MA, Açailândia*; PA, Parauapebas \{Serra Norte, Carajás\}*, Santarém*; RO, Cacaulândia*, Porto Velho $\{$ Aliança\} (Rothschild 1910)

AM, Fonte Boa (Rothschild 1909a); RO, Cacaulândia*

AP, Serra do Navio*; MA, Açailândia*; PA, Santarém (Dognin 1923c); RO, Cacaulândia*

PA, Prainha (Butler 1877)

AM, Manaus*, São Paulo de Olivença*; MA, Açailândia*; PA, Altamira \{Serra do Pardo National Park\} (Teston and Correa 2015), [Belém] (Rothschild 1922), Marabá*, São Félix do Xingu \{Serra do Pardo National Park\} (Teston and Correa 2015); RO, Cacaulândia*

AM, Fonte Boa (Rothschild 1909a); RO, Porto Velho \{Calama\} (Rothschild 1910)

AM, Codajás (Rothschild 1910), Fonte Boa (Rothschild 1910), Moura*, São Gabriel da Cachoeira \{Querari\}*, Tefé (Rothschild 1910); AP, Serra do Navio*; PA, Almeirim \{Jari\} (Hawes et al. 2009), [Belém] (Walker 1856), São Félix do Xingu \{Serra do Pardo National Park\} (Teston and Correa 2015), São Francisco do Pará \{Anhanga\}*; RO, Cacaulândia*, Porto Velho*

AM, Fonte Boa (Rothschild 1909a), Moura*; MT, Sinop*; PA, [Belém] (Rothschild 1922); RO, Candeias do Jamari* AM, Fonte Boa (Rothschild 1909a), Tefé (Seitz 1921)

AM, Fonte Boa(Rothschild 1909a)

AM, Atalaia do Norte \{Santo Antonio do Javari\} (Rothschild 1909a); PA, [Belém] (Rothschild 1922), São Félix do Xingu \{Serra do Pardo National Park\} (Teston and Correa 2015); RO, Porto Velho*

AM, Atalaia do Norte \{Santo Antonio do Javari\} (Rothschild 1910), Benjamin Constant*, Fonte Boa (Rothschild 1910), Manaus*, São Paulo de Olivença (Hampson 1901), Tefé (Rothschild 1910); AP, Serra do Navio*; MT, Sinop*; PA, [Belém] (Rothschild 1922), Gurupá*, Marabá*, Parauapebas \{Serra Norte, Carajás\} ${ }^{*}$, São Félix do Xingu \{Serra do Pardo National Park\} (Teston and Correa 2015), Viseu*; RO, Cacaulândia*, Candeias do Jamari*, Porto Velho \{Aliança\} (Rothschild 1910)

AM, Benjamin Constant*, Fonte Boa (Rothschild 1909a); AP, Serra do Navio*; RO, Cacaulândia*, Candeias do Jamari*

AM, Fonte Boa (Rothschild 1910), Tefé (Rothschild 1910)

AM, Benjamin Constant*

RR, Pacaraima* 
Table 1. Continued.

Species
Psychophasma erosa (Herrich-Schäffer, [1858])

Purius pilumnia (Stoll, [1780])

Pydnaodes distincta (Rothschild, 1909) Regobarrosia flavescens (Walker, 1856) Rhipha albiplaga (Schaus, 1905)

Rhipha flammans (Hampson, 1901) Rhipha luteoplaga (Rothschild, 1922) Rhipha niveomaculata (Rothschild, 1909) Rhipha perflammans (Dognin, 1914) NEW Rhipha persimilis (Rothschild, 1909) Rhipha strigosa (Walker, 1854)

Rhipha subflammans (Rothschild, 1909) AMz Robinsonia boliviana Seitz, 1921 Robinsonia dewitzi Gundlach, 1881

\section{Robinsonia exprata (Dognin, 1921)} Robinsonia flavomarginata Druce, 1899 Robinsonia fogra Schaus, 1895 Robinsonia klagesi Rothschild, $1910^{\text {NEW }}$ Robinsonia lefaivrei Schaus, 1895

Robinsonia marginata Rothschild, 1909 NEW Robinsonia morula Druce, 1906 Robinsonia mossi (Rothschild, 1922) Robinsonia praphoea Dognin, 1906 Robinsonia rockstonia Schaus, 1905

Robinsonia sanea Druce, 1895

Robinsonia suffusa Rothschild, 1909 Scaptius asteroides (Schaus, 1905)

Scaptius chrysopera (Schaus, 1905)

Scaptius ditissimus Walker, 1855 Scaptius obscurata (Schaus, 1920) NEW Scaptius prumaloides (Rothschild, 1909) Scaptius sordida (Rothschild, 1935) Scaptius submarginalis (Rothschild, 1909) Selenarctia elissa (Schaus, 1892)

Selenarctia elissoides (Rothschild, 1909) NEW Stidzaeras strigifera Druce, 1905

Sutonocrea lobifer (H. Schaeffer, [1855]) Sutonocrea reducta (Walker, 1856)

Sychesia dryas (Cramer, [1776])

Sychesia melini Bryk, 1953

Sychesia omissus (Rothschild, 1910)

\section{Record}

AM, Alto [Rio] Solimões (Rego Barros 1946), Benjamin Constant \{Rio Quichito\} (Rego Barros 1946), Fonte Boa*, Humaitá \{Rio Madeira\} (Rothschild 1910), Itacoatiara*, Manaus*, Rio Juruá (Butler 1878), Rio Negro (Rothschild 1910), Rio Prato (Rego Barros 1946), [São Gabriel da Cachoeira] \{Taracuá\} (Bryk 1953), São Paulo de Olivença (Rego Barros 1946), Tefé (Walker [1865]); AP, Serra do Navio*; MA, Açailândia*; MT, Sinop*; PA, Almeirim \{Jari\} (Hawes et al. 2009), Altamira \{Monte Santo\} (Teston and Delfina 2010), [Belém] (Rothschild 1922), Marabá*, Novo Progresso $\left\{\right.$ Cachimbo\}* ${ }^{*}$ Parauapebas $\left\{\right.$ Serra Norte, Carajás\} ${ }^{*}$, Santarém*, São Félix do Xingu $\{$ Serra do Pardo National Park\} (Teston and Correa 2015), Tucuruí; ; RO, Cacaulândia*, Porto Velho \{Calama\} (Rothschild 1910); RR, Alto Alegre*

AM, [Fonte Boa] ([Lago] Uará) (Travassos 1951a), Humaitá*, Manaus (Hampson 1901), Manicoré \{Rio Madeira\} (Travassos 1951a), Uypiranga \{Rio Madeira\} (Travassos 1951a), São Paulo de Olivença (Travassos 1951a); PA, Anajás*, [Belém] (Walker 1855), Santarém*

AM, Fonte Boa (Rothschild 1909c)

PA, [Belém] (Walker 1856), São Félix do Xingu \{Serra do Pardo National Park\} (Teston and Correa 2015)

AM, Fonte Boa (Rothschild 1910), São Paulo de Olivença*; AP, Serra do Navio*; MT, Sinop*; PA, Altamira and São Félix do Xingu \{Serra do Pardo National Park\} (Teston and Correa 2015); RO, Cacaulândia*, Candeias do Jamari* AM, Borba*; AP, Serra do Navio*; MA, Açailândia*; PA, [Belém] (Rothschild 1922); RO, Cacaulândia*

PA, [Belém] (Rothschild 1922)

PA, [Belém] (Rothschild 1922)

MA, Açailândia*

AM, Codajás (Rothschild 1910), Fonte Boa (Rothschild 1909a)

AM, Fonte Boa (Rothschild 1910), Itacoatiara*, São Paulo de Olivença (Travassos 1943); PA, [Belém] (Rothschild 1922), Parauapebas \{Serra Norte, Carajás\}; ${ }^{*}$ RO, Cacaulândia* ${ }^{*}$ Porto Velho*

RR, Alto Alegre*

AP, Serra do Navio*; MA, Açailândia*; PA, Altamira \{Monte Santo\} (Teston and Delfina 2010); RO, Cacaulândia*

AP, Serra do Navio*; MA, Açailândia*; PA, [Belém] (Rothschild 1922), São Félix do Xingu \{Serra do Pardo National Park\} (Teston and Correa 2015); RO, Cacaulândia*; RR, Alto Alegre*

PA, [lgarapé Açu] \{Prata 100 kil[ometers] de Pará\} (Dognin 1921)

[AM], [Rio] Amazonas (Rothschild 1910); AP, Serra do Navio*

PA, Almeirim \{Jari\} (Hawes et al. 2009); RO, Cacaulândia*

AP, Serra do Navio*; MA, Açailândia*; RO, Cacaulândia*, Candeias do Jamari*, Porto Velho*

AM, Benjamin Constant \{Rio Quichito\} (Travassos 1964), Fonte Boa (Rothschild 1910); AP, Serra do Navio (Travassos 1964); PA, Marabá*; RO, Cacaulândia*

AP, Serra do Navio*

AM, Fonte Boa (Rothschild 1910); RO, Jaru*, Porto Velho (Travassos 1964)

MA, Açailândia*; PA, [Belém] (Rothschild 1922)

AM, Atalaia do Norte \{Santo Antonio do Javari\} (Rothschild 1910), Fonte Boa (Rothschild 1910)

AM, Fonte Boa (Rothschild 1910); AP, Serra do Navio*; PA, Altamira and São Félix do Xingu \{Serra do Pardo National Park\} (Teston and Correa 2015); RO, Cacaulândia*

MA, Açailândia*; PA, Marabá*, São Félix do Xingu \{Serra do Pardo National Park\} (Teston and Correa 2015); [PA], Baixo [Rio] Amazonas (Rothschild 1910)

AM, Fonte Boa (Rothschild 1909a)

AP, Serra do Navio*; PA, São Félix do Xingu \{Serra do Pardo National Park\} (Teston and Correa 2015); RO, Cacaulândia*

AM, Fonte Boa*; AP, Serra do Navio*; MA, Açailândia*; PA, São Félix do Xingu \{Serra do Pardo National Park\} (Teston and Correa 2015); RO, Cacaulândia*

AM, Tefé (Walker 1855)

MA, Açailândia*

AM, Fonte Boa (Rothschild 1909a)

PA, [Belém] (Rothschild 1935)

PA, [Belém] (Rothschild 1922), Marabá*

AM, Atalaia do Norte \{Santo Antonio do Javari\} (Rothschild 1910), Fonte Boa (Rothschild 1910); PA, [Belém] (Rothschild 1922), Parauapebas \{Serra Norte, Carajás\}*, Óbidos \{Curumucury\} (Watson 1975), São Félix do Xingu \{Serra do Pardo National Park\} (Teston and Correa 2015)

AM, Moura*

PA, Altamira \{Serra do Pardo National Park\} (Teston and Correa 2015), Belém*, Parauapebas \{Serra Norte, Carajás\}*; RO, Cacaulândia*

AM, Benjamin Constant (Travassos 1944c), Fonte Boa (Rothschild 1910); AP, Serra do Navio*

AM, Fonte Boa*, Manaus (Bryk 1953), Moura*, Vale [do Rio] Amazonas (Walker 1856); AP, Serra do Navio*; MT, Sinop*; PA, [Belém] (Rothschild 1922), Igarapé-Açu*, Parauapebas \{Serra Norte, Carajás\}*, Santarém*, São Félix do Xingu \{Serra do Pardo National Park\} (Teston and Correa 2015); RO, Cacaulândia*

AM, Fonte Boa (Rothschild 1910), [Jutaí] \{Rio Sapo\} (Hampson 1901), Manacapuru to Tefé (Rothschild 1910); PA, [Belém] (Walker 1856)

AM, [Rio] Solimões (Bryk 1953), Tabatinga (Bryk 1953)

AM, Atalaia do Norte \{Santo Antonio do Javari\} (Rothschild 1910), Fonte Boa (Rothschild 1910); RO, Porto Velho \{Calama\} (Rothschild 1910) 
Table 1. Continued.

\begin{tabular}{l}
\hline Species \\
\hline Sychesia subtilis (Butler, 1878) \\
Symphlebia favillacea (Rothschild, 1909) \\
Symphlebia hyalina (Rothschild, 1909) \\
Symphlebia lophocampoides Felder \& \\
Rogenhofer, $1874{ }^{\text {AMz }}$ \\
Symphlebia muscosa (Schaus, 1910) NEW \\
Symphlebia neja (Schaus, 1905)
\end{tabular}

Syntomostola semiflava Dognin, 1923 Tessella klagesi (Rothschild, 1909)

Thyromolis pythia (Druce, 1900)

Toulgoetarctia haematora Coenen \& Gibeaux, 2014

Toulgoetarctia sanguinea (Hampson, 1905)

Trichromia albicollis (Hampson, 1905)

Trichromia androconiata (Rothschild, 1909) Trichromia atta (Schaus, 1920) NEW Trichromia aurantiipennis (Rothschild, 1909) Trichromia cardinalis (Dognin, 1899) NEW Trichromia carinaria (Schaus, 1905) Trichromia carminata (Schaus, 1905) Trichromia cinerea (Rothschild, 1913) Trichromia clarivena (Gaede, 1923) Trichromia coccinea (Schaus, 1905) Trichromia coccineothorax (Rothschild, 1922) Trichromia complicata (Schaus, 1905) NEW Trichromia cucufas (Schaus, 1924) NEW Trichromia cuneoplagiatus (Rothschild, 1922) Trichromia cyclopera (Hampson, 1905)

Trichromia declivis (Schaus, 1905)

Trichromia discobola (Hampson, 1905) Trichromia discophora Hampson, 1916 NEW Trichromia flavimargo (Joicey \& Talbot, 1916) NEW Trichromia flavomarginata (Rothschild, 1910) Trichromia flavoroseus (Walker, 1855) Trichromia furva (Schaus, 1905) Trichromia gaudialis (Schaus, 1905) Trichromia griseata (Rothschild, 1909) Trichromia impunctata (Gaede, 1928) Trichromia incerta (Schaus, 1905) Trichromia inequalis (Rothschild, 1909)

Trichromia interna (Schaus, 1905) NEW Trichromia leucoplaga (Hampson, 1905) Trichromia lucens (Schaus, 1905) NEW Trichromia maculata (Rothschild, 1909) Trichromia macrostidza (Hampson, 1905) Trichromia mathani (Rothschild, 1909) Trichromia metachryseis (Hampson, 1905) Trichromia metapyria (Dognin, 1907) Trichromia methaemia (Schaus, 1905) Trichromia odorata (Rothschild, 1909) Trichromia onytes (Cramer, [1777])

Trichromia pandera Schaus, 1896 Trichromia patara (Druce, 1896) Trichromia persimilis (Rothschild, 1909) Trichromia perversa (Rothschild, 1909)
Record

AM, [Jutaí] \{Rio Jutahi above Rio Curmen, Rio Sapo\} (Butler, 1878), [Manaus] \{[llha] Marapatá\} (Butler 1878)

PA, [Belém] (Rothschild 1922)

AM, Fonte Boa (Rothschild 1910), São Paulo de Olivença*; PA, [Belém] (Rothschild 1922); RO, Porto Velho* AM, Manaus*

AP, Serra do Navio*

AM, Fonte Boa (Rothschild 1910), Moura*, São Gabriel da Cachoeira \{Querari\}*; AP, Serra do Navio*; PA, [Belém] (Rothschild 1922), Novo Progresso \{Cachimbo\}*; RO, Porto Velho*

AM, São Paulo de Olivença (Dognin 1923a)

AM, Fonte Boa (Rothschild 1909c); PA, [Belém] (Rothschild 1922)

AM, Benjamin Constant* Fonte Boa (Rothschild 1910), São Gabriel de Cahoeira \{Marabitanas\}*; MA, Açailândia*; RO, Cacaulândia*

AM, Fonte Boa (Rothschild 1910)

PA, [Belém] (Rothschild 1922)

AM, Benjamin Constant* Fonte Boa (Rothschild 1909b), Manaus*, São Gabriel da Cachoeira \{Querari\}*; AP, Serra do Navio*; MA, Açailândia*; MT, Sinop*; PA, Capitão Poço*, Marabá*, São Félix do Xingu \{Serra do Pardo National Park\} (Teston and Correa 2015); RO, Cacaulândia*, Porto Velho*

AM, Benjamin Constant*, Fonte Boa (Rothschild 1910); PA, Almeirim \{Jari\} (Hawes et al. 2009); RO, Cacaulândia* RO, Porto Velho*

AM, Fonte Boa (Rothschild 1909c); PA, Marabá*

AP, Amapari*; PA, Marabá*; RO, Cacaulândia*

PA, Altamira \{Monte Santo\} (Teston and Delfina 2010)

PA, [Belém] (Rothschild 1922)

AM, Fonte Boa (Rothschild 1913)

AM, São Paulo de Olivença (Gaede 1923)

AM, Fonte Boa (Rothschild 1910)

PA, [Belém] (Rothschild 1922)

RO, Cacaulândia*

AM, Benjamin Constant*; RO, Ariquemes*, Cacaulândia*, Porto Velho*

PA, [Belém] (Rothschild 1922), Santarém (Vincent and Laguerre 2014)

AM, Fonte Boa (Rothschild 1910); PA, Altamira \{Monte Santo\} (Teston and Delfina 2010), [Belém] (Rothschild 1922)

MA, Açailândia*; PA, Almeirim \{Jari\} (Hawes et al. 2009), Belém*, Igarapé-Açu*, Marabá*, São Félix do Xingu \{Serra do Pardo National Park\} (Teston and Correa 2015); RO, Porto Velho*

AM, Atalaia do Norte \{Santo Antonio do Javari\} (Rothschild 1910), Fonte Boa (Rothschild 1910)

AM, Benjamin Constant*, Fonte Boa*; AP, Serra do Navio*; PA, Belém*

PA, Marabá*; RO, Ariquemes*

AM, Fonte Boa (Rothschild 1910)

PA, [Belém] (Walker 1855)

PA, [Belém] (Rothschild 1922)

AP, Serra do Navio*; PA, [Belém] (Rothschild 1922), Marabá*

AM, Borba*, Fonte Boa (Rothschild 1909a)

PA, Juruti (Gaede 1928)

AM, São Gabriel da Cachoeira \{Querari\}*; AP, Serra do Navio*; PA, [Belém] (Rothschild 1922)

AM, Codajás (Rothschild 1909b), Manaus*, Fonte Boa (Rothschild 1909b); AP, Serra do Navio*; MA, Açailândia*;

PA, Marabá*; RO, Cacaulândia*

AP, Amapari*, Serra do Navio*; MA, Açailândia*; PA, Belém*; RO, Cacaulândia*

AM, Manaus*; AP, Serra do Navio*; MA, Açailândia*; PA, [Belém] (Rothschild 1922); RO, Cacaulândia*

AM, Manaus*; AP, Serra do Navio*; PA, Belém*, Viseu*; RO, Cacaulândia*

AM, Fonte Boa (Rothschild 1909a), Manaus*

AM, Fonte Boa (Rothschild 1910), [Jutaí] \{Rio Jutai\} (Hampson 1901); PA, [Belém] (Hampson 1901)

AM, Tefé (Rothschild 1909b); PA, Marabá*; RO, Ariquemes*, Cacaulândia*, Porto Velho*

PA, São Félix do Xingu \{Serra do Pardo National Park\} (Teston and Correa 2015)

PA, [Belém] (Rothschild 1922), São Félix do Xingu \{Serra do Pardo National Park\} (Teston and Correa 2015)

PA, [Belém] (Rothschild 1922)

AM, Fonte Boa (Rothschild 1909c)

AM, Borba*; PA, [Belém] (Rothschild 1922), São Félix do Xingu \{Serra do Pardo National Park\} (Teston and Correa 2015); RO, Cacaulândia*

AM, Benjamin Constant*, Rio Negro (Hampson 1901); RO, Cacaulândia*

AM, Fonte Boa (Rothschild 1909a); MA, Açailândia*; MT, Sinop*; PA, Marabá*; RO, Cacaulândia*

AM, Codajás (Rothschild 1910), Fonte Boa (Rothschild 1909b); PA, Almeirim \{Jari\} (Hawes et al. 2009) AM, Fonte Boa (Rothschild 1909b) 
Table 1. Continued.

\begin{tabular}{l}
\hline Species \\
\hline Trichromia phaeoplaga (Hampson, 1905) NEW \\
Trichromia polyxenoides (Rothschild, 1909) \\
Trichromia polyxenus (Druce, 1883) NEW \\
Trichromia postsuffusa (Rothschild, 1922) \\
Trichromia psamas (Cramer, [1779])
\end{tabular}

Trichromia purpurascens (Rothschild, 1909) Trichromia repanda (Walker, 1855) Trichromia rosacea (Rothschild, 1909) Trichromia rosaceata (Watson \& Goodger, 1986) Trichromia sanguipuncta (Schaus, 1901) NEW Trichromia sardanapalus (Rothschild, 1909) Trichromia sithnides (Rothschild, 1909) Trichromia sorex (Druce, 1902)

Trichromia tipolis (Druce, 1896) Trichromia trilobata (Gaede, 1923) Trichromia viola (Dognin, 1909) Tricypha furcata Möschler, 1878

Tricypha imperialis (Heylaerts, 1884)

Tricypha pseudotricypha (Rothschild, 1909) Viviennea dolens (Druce, 1904) AMz Viviennea flavicincta (Herrich-Schäffer, [1855]) Viviennea gyrata (Schaus, 1920) Viviennea moma (Schaus, 1905)

Viviennea salma (Druce, 1896) NEW Viviennea superba (Druce, 1883)

Viviennea zonana (Schaus, 1905) Xanthoarctia pseudameoides (Rothschild, 1909)

Zaevius calocore Dyar, 1910 Zatrephes albescens Rothschild, 1909 Zatrephes amoena Dognin, 1924 Zatrephes arenosa Schaus, 1905 NEW Zatrephes bicolorata (Druce, 1906) NEW Zatrephes bilineata rufobrunnea Rothschild, 1909

Zatrephes binotata Rothschild, 1909

Zatrephes cardytera Dyar, 1910

Zatrephes carmesina (Rothschild, 1909)

Zatrephes crocos (Cramer, [1777])

Zatrephes cruciata Rothschild, 1909 Zatrephes fallax Dognin, 1923 Zatrephes fasciola Seitz, 1922 Zatrephes flavida Hampson, 1905 Zatrephes foliacea Rothschild, 1909 Zatrephes flavipuncta Rothschild, 1909 Zatrephes gigantea Rothschild, 1909 Zatrephes griseorufa Rothschild, 1909 Zatrephes iridescens iridescens Rothschild, 1910 Zatrephes irrorata Rothschild, 1909 Zatrephes klagesi Rothschild, 1909 Zatrephes krugeri Reich, 1934
Record

PA, Belém*; RO, Cacaulândia*

AM, Fonte Boa (Rothschild 1909b); PA, [Belém] (Rothschild 1922)

AP, Serra do Navio*; MA, Açailândia*; PA, Marabá*

PA, [Belém] (Rothschild 1922)

MA, Açailândia*; PA, [Belém] (Rothschild 1922), Capitão Poço*, Marabá*; RO, Cacaulândia*, Porto Velho \{Calama\} (Rothschild 1910)

AM, Fonte Boa (Rothschild 1909c); PA, Marabá*

AM, Fonte Boa (Rothschild 1910); AP, Serra do Navio*; PA, Belém*

AM, Fonte Boa (Rothschild 1909b)

PA, [Belém] (Rothschild 1935)

MA, Açailândia*; RO, Cacaulândia*

AM, Fonte Boa (Rothschild 1909a); PA, [Belém] (Rothschild 1922)

AM, Fonte Boa (Rothschild 1909b); PA, [Belém] (Rothschild 1922); RO, Cacaulândia*, Porto Velho*

AM, Fonte Boa (Rothschild 1910), Rio Autaz (Bryk 1953); PA, Almeirim \{Jari\} (Hawes et al. 2009), [Belém] (Rothschild 1922); RO, Cacaulândia*

PA, Almeirim \{Jari\} (Hawes et al. 2009)

AM, São Paulo de Olivença (Gaede 1923)

AM, Rio Madeira (Rothschild 1922); MA, Açailândia*; PA, [Belém] (Rothschild 1922), Marabá*; RO, Cacaulândia* AM, Codajás (Rothschild 1910), Fonte Boa (Rothschild 1910), Lábrea \{Rio Purus, [S]epatiny\} (Butler 1878), Rio Purus \{Araras\} (Butler 1878); RO, Porto Velho \{Aliança\} (Rothschild 1910)

AM, Rio Negro (Hampson 1901), São Paulo de Olivença*, Barcelos \{Tomar\} (Hampson 1901); PA, Santarém (Hampson 1901)

AM, Fonte Boa (Rothschild 1910)

RO, Porto Velho*

[AM], [Rio] Amazon[a]s (Kirby 1892)

PA, Almeirim \{Jari\} (Hawes et al. 2009)

AM, Atalaia do Norte \{Santo Antonio do Javari\} (Rothschild 1910), Benjamin Constant* , Fonte Boa (Rothschild 1910), Lábrea \{Huitanaã - Rio Purus\} (Watson 1975), Manaus*, São Paulo de Olivença (Watson 1975), Tefé (Rothschild 1910); AP, Serra do Navio*; MA, Açailândia*; PA, [Belém] (Rothschild 1922), Novo Progresso $\left\{\right.$ Cachimbo\}* ${ }^{*}$ Parauapebas \{Serra Norte, Carajás\}* , [Rio Tocantins] (Watson 1975), São Félix do Xingu \{Serra do Pardo National Park\} (Teston and Correa 2015); RO, Cacaulândia*, Candeias do Jamari*, Jaru*, Porto Velho (Watson 1975)

AM, Benjamin Constant*, Manaus*; MA, Açailândia*; PA, Tucuruí;; RO, Cacaulândia*

AM, Fonte Boa (Rothschild 1910), Lábrea \{Huitanaã - Rio Purus\} (Watson 1975), Nova Olinda \{Rio Purus\} (Watson 1975), São Paulo de Olivença (Watson 1975), Tefé (Watson 1975); PA, [Belém] (Rothschild 1922), São Félix do Xingu \{Serra do Pardo National Park\} (Teston and Correa 2015)

AM, Lábrea \{Huitanaã - Rio Purus\} (Watson 1975); RO, Cacaulândia*

AM, Benjamin Constant \{Rio Quichito\} (Travassos 1951c), Fonte Boa (Rothschild 1910), Manaus \{Uypiranga\} (Travassos 1951c), Manicoré (Travassos 1951c), São Gabriel da Cachoeira \{Querari\}*; AP, Serra do Navio*; MT, Sinop*; RO, Candeias do Jamari*, Porto Velho*

AM, Fonte Boa (Rothschild 1922); PA, [Belém] (Rothschild 1922)

AM, Fonte Boa (Rothschild 1909a)

AM, São Paulo de Olivença (Dognin 1924)

RO, Cacaulândia*

AM, Benjamin Constant*, Fonte Boa*

AM, Benjamin Constant ${ }^{*}$, Fonte Boa (Rothschild 1909a); PA, [Belém] (Rothschild 1922)

AM, Fonte Boa (Rothschild 1909a)

PA, Altamira \{Monte Santo\} (Teston and Delfina 2010)

AM, Fonte Boa (Rothschild 1909a)

AM, Fonte Boa (Rothschild 1910), Tefé (Rothschild 1910); PA, São Félix do Xingu \{Serra do Pardo National Park\} (Teston and Correa 2015)

AM, Fonte Boa (Rothschild 1909a)

AM, Manaus*, São Paulo de Olivença (Dognin 1923c)

AM, Manaus*, Moura*, São Paulo de Olivença*, Tefé (Seitz 1922); RO, Cacaulândia*, Candeias do Jamari*

AM, Fonte Boa (Rothschild 1910); PA, [Belém] (Rothschild 1922)

AM, Fonte Boa (Rothschild 1909a); RO, Porto Velho \{Calama\} (Rothschild 1910)

AM, Fonte Boa (Rothschild 1910)

AM, Fonte Boa (Rothschild 1909a)

AM, Fonte Boa (Rothschild 1909a)

AM, Fonte Boa (Rothschild 1909a); RO, Porto Velho*

AM, Fonte Boa (Rothschild 1909a)

AM, Fonte Boa (Rothschild 1909a)

AM, São Paulo de Olivença (Reich 1934) 
Table 1. Continued.

\section{Species}

Zatrephes magnifenestra Bryk, 1953

Zatrephes miniata Rothschild, 1909

Zatrephes modesta Schaus, 1905

Zatrephes mossi Rothschild, 1933

Zatrephes nitida (Cramer, [1780])

Zatrephes ockendeni Rothschild, 1909 NEW

Zatrephes olivenca Dognin, 1923

Zatrephes ossea Schaus, 1905

Zatrephes propinqua (Rothschild, 1909) NEW

Zatrephes rosacea Rothschild, 1909

Zatrephes rufescens Rothschild, 1909

Zatrephes subflavescens Rothschild, 1909

Zatrephes trailii Butler, 1877

Zatrephes trilineata trilineata Hampson, 1905

Zatrephes variegata Rothschild, 1909
Record

AM, Rio Purus (Bryk 1953)

AM, Atalaia do Norte \{Santo Antonio do Javari\} (Rothschild 1910), Benjamin Constant*, Fonte Boa (Rothschild 1909a)

PA, [Belém] (Rothschild 1922)

PA, [Belém] (Rothschild 1933)

AM, Benjamin Constant*, Fonte Boa (Rothschild 1910), Manaus*, São Paulo de Olivença*; AP, Serra do Navio*; PA, [Belém] (Rothschild 1922); RO, Porto Velho*

RO, Porto Velho*

AM, São Paulo de Olivença (Dognin 1923a)

AM, Fonte Boa (Rothschild 1910)

AM, Fonte Boa*

AM, Benjamin Constant*, Fonte Boa (Rothschild 1909a), São Paulo de Olivença*, Tefé (Rothschild 1910); PA, [Belém] (Rothschild 1922); RO, Porto Velho*

AM, Atalaia do Norte \{Santo Antonio do Javari\} (Rothschild 1909a), Benjamin Constant* Fonte Boa (Rothschild 1909a); PA, [Belém] (Rothschild 1922)

AM, Atalaia do Norte \{Santo Antonio do Javari\} (Rothschild 1910), Fonte Boa (Rothschild 1909a)

AM, Benjamin Constant*, Fonte Boa (Rothschild 1910), Manaus*, Rio Juruá (Butler 1877), São Paulo de Olivença*; AP, Serra do Navio*; PA, [Belém] (Rothschild 1922)

AM, Benjamin Constant*, Fonte Boa (Rothschild 1910); PA, [Belém] (Rothschild 1922) AM, Fonte Boa (Rothschild 1909a); RO, Porto Velho*

AMz Species recorded for the Amazon biome by Ferro and Diniz (2010), but without accurate location information. So these species are new records for the municipalities and their respective States. NEW New record for the Brazilian Amazon. 